\title{
Travelling waves and a fruitful 'time' reparametrization in relativistic electrodynamics
}

\author{
Gaetano Fiore \\ Dip. di Matematica e Applicazioni, Università di Napoli "Federico II", \\ \& INFN, Sez. di Napoli, \\ Complesso Universitario M. S. Angelo, Via Cintia, 80126 Napoli, Italy
}

\begin{abstract}
We simplify the nonlinear equations of motion of charged particles in an external electromagnetic field that is the sum of a plane travelling wave $F_{t}^{\mu \nu}(c t-z)$ and a static part $F_{s}^{\mu \nu}(x, y, z)$ : by adopting the light-like coordinate $\xi=c t-z$ instead of time $t$ as an independent variable in the Action, Lagrangian and Hamiltonian, and deriving the new Euler-Lagrange and Hamilton equations accordingly, we make the unknown $z(t)$ disappear from the argument of $F_{t}^{\mu \nu}$. We study and solve first the single particle equations in few significant cases of extreme accelerations. In particular we obtain a rigorous formulation of a Lawson-Woodward-type (no-final-acceleration) theorem and a compact derivation of cyclotron autoresonance, beside new solutions in the presence of uniform $F_{s}^{\mu \nu}$. We then extend our method to plasmas in hydrodynamic conditions and apply it to plane problems: the system of (Lorentz-Maxwell+continuity) partial differential equations may be partially solved or sometimes even completely reduced to a family of decoupled systems of ordinary ones; this occurs e.g. with the impact of the travelling wave on a vacuum-plasma interface (what may produce the slingshot effect).

Our method can be seen as an application of the light-front approach. Since Fourier analysis plays no role in our general framework, the method can be applied to all kind of travelling waves, ranging from almost monochromatic to socalled "impulses", which contain few, one or even no complete cycle.
\end{abstract}

\section{Introduction}

In the general form the equation of motion of a charged particle in an external electromagnetic field $F^{\mu \nu}=\partial^{\mu} A^{\nu}-\partial^{\nu} A^{\mu}$ is non-autonomous and highly nonlinear in the unknowns $\boldsymbol{x}(t), \boldsymbol{p}(t)$ :

$$
\begin{aligned}
& \dot{\boldsymbol{p}}(t)=q \boldsymbol{E}[c t, \boldsymbol{x}(t)]+\frac{\boldsymbol{p}(t)}{\sqrt{m^{2} c^{2}+\boldsymbol{p}^{2}(t)}} \wedge q \boldsymbol{B}[c t, \boldsymbol{x}(t)], \\
& \dot{\boldsymbol{x}}(t)=\frac{c \boldsymbol{p}(t)}{\sqrt{m^{2} c^{2}+\boldsymbol{p}^{2}(t)}}
\end{aligned}
$$


Here $m, q, \boldsymbol{x}, \boldsymbol{p}$ are the rest mass, electric charge, position and relativistic momentum of the particle, $\boldsymbol{E}=-\partial_{t} \boldsymbol{A} / c-\nabla A^{0}$ and $\boldsymbol{B}=\nabla \wedge \boldsymbol{A}$ are the electric and magnetic field, $\left(A^{\mu}\right)=\left(A^{0},-\boldsymbol{A}\right)$ is the electromagnetic (EM) potential 4 -vector $\left(E^{i}=F^{i 0}, B^{1}=F^{32}\right.$, etc.; we use Gauss CGS units). Usually, the analytical study of (1) is somewhat simplified under one or more of the following physically relevant conditions: $F^{\mu \nu}$ are constant (i.e. static and uniform EM field) or vary "slowly" in space or time; $F^{\mu \nu}$ are "small" (so that nonlinear effects in the amplitudes are negligible); $F^{\mu \nu}$ are monochromatic waves or slow modulations of the latter; the motion remains non-relativistic. ${ }^{1}$ The amazing developments of laser technologies (especially chirped pulse amplification $[5,6,7]$ ) have made available compact sources of extremely intense (up to $10^{23} \mathrm{~W} / \mathrm{cm}^{2}$ ) coherent EM waves; the latter can be also concentrated in very short laser pulses (tens of femtoseconds), or superposed to very strong static EM fields. Even more intense and short laser pulses will be produced in the near future through new technologies (thin film compression, relativistic mirror compression, coherent amplification networks $[8,9])$. One of the main motivation behind these developments is the enhancement of the Laser Wake Field Acceleration (LWFA) mechanism ${ }^{2}$ [10, 11, 12], with a host of important applications (ranging from cancer therapy, to X-ray free electron laser, radioisotope production, high energy physics, etc.; see e.g. [13, 9] for reviews). Extreme conditions occur also in a number of violent astrophysical processes (see e.g. [9] and references therein). The interaction of isolated electric charges or continuous matter with such coherent waves (and, possibly, static EM fields) is characterized by so fast, huge, highly nonlinear and ultra-relativistic effects that the mentioned simplifying conditions are hardly fulfilled, and the standard approximation schemes are seriously challenged. Alternative approaches are therefore desirable.

Here we develop an approach that is especially fruitful when the wave part of the EM field can be idealized as an external plane travelling wave $F_{t}^{\mu \nu}(c t-z)$ (where $\boldsymbol{x}=x \mathbf{i}+y \mathbf{j}+z \mathbf{k}$, with suitable cartesian coordinates) in the spacetime-region $\Omega$ of interest (i.e., where we are interested to follow the worldlines of the charged particles). This requires that the initial wave be of this form and radiative corrections, curvature of the front, diffraction effects be negligible in $\Omega$. Normally these conditions can be fulfilled in vacuum; sometimes also in low density matter (even in the form of a plasma, see section 4) for short times after the beginning of the interaction with the wave. ${ }^{3}$ The starting point is the (rather obvious) observation that, since no particle can reach the speed of light, the function $\tilde{\xi}(t)=c t-z(t)$ is strictly growing and therefore we can adopt $\xi=c t-z$ as a parameter on the worldline of the particle. Integrating over $\xi$ in the particle action functional, applying Hamilton's principle and the Lejendre transform we thus find Lagrange and Hamilton equations with $\xi$ as the

\footnotetext{
${ }^{1}$ In particular, standard textbooks of classical electrodynamics like [1, 2, 3] discuss the solutions only under a constant or a slowly varying (in space or time) $F^{\mu \nu}$; in [4] also under an arbitrary purely transverse wave (see section 3.1), or a Coulomb electrostatic potential.

${ }^{2}$ In the LWFA laser pulses in a plasma produce plasma waves (i.e. waves of huge charge density variations) via the ponderomotive force (see section 3.1); these waves may accelerate electrons to ultrarelativistic regimes through extremely high acceleration gradients (such as $1 \mathrm{GV} / \mathrm{cm}$, or even larger).

${ }^{3}$ Causality helps in the fulfillment of these requirements: We can assign the initial conditions for the system of dynamic equations on the $t=t_{0}$ Cauchy hyperplane $S_{t_{0}}$, where $t_{0}$ is the time of the beginning of wave-matter interaction. In a sufficiently small region $\mathcal{D}_{\boldsymbol{x}} \subset \mathrm{S}_{t_{0}}$ around any point $\boldsymbol{x}$ of the wave front the EM wave is practically indistinguishable from a plane one $F_{t}$. Therefore the solutions induced by the real wave and by its plane idealization $F_{t}$ will be practically indistinguishable within the future Cauchy development $D^{+}\left(\mathcal{D}_{\boldsymbol{x}}\right)$ of $\mathcal{D}_{\boldsymbol{x}}$.
} 
independent variable. Since the unknown $\hat{\boldsymbol{x}}(\xi)=\boldsymbol{x}(t)$ no more appears in the argument of the wave part $F_{t}$ of the EM field

$$
\hat{F}^{\mu \nu}(\xi, \hat{\boldsymbol{x}})=F_{t}^{\mu \nu}(\xi)+F_{s}^{\mu \nu}(\hat{\boldsymbol{x}}),
$$

$F_{t}(\xi)$ acts as a known forcing term, and these new equations are simpler than the usual ones, where the unknown combination $c t-z(t)$ appears as the argument in $F_{t}^{\mu \nu}[c t-z(t)]$. The light-like relativistic factor $s=d \xi / d(c \tau)$ (light-like component of the momentum, in normalized units) plays the role of the Lorentz relativistic factor $\gamma=d t / d \tau$ in the usual formulation and has remarkable properties: all 4-momentum components are rational functions of it and of the transverse momentum; if the static electric and magnetic fields have only longitudinal components then $s$ is practically insensitive to fast oscillation of $F_{t} . s$ was introduced somehow ad hoc in [?, 15] (see also [16, 17]); here we clarify its meaning and role. We shall see that the dependence of the dynamical variables on $\xi$ allows a more direct determination of a number of useful quantities (like the momentum, energy gain, etc) of the particle, either in closed form or by numerical resolution of the simplified differential equations; their dependence on $t$ can be of course recovered after determining $\hat{z}(\xi)$.

The use of a light-like coordinate instead of $t$ as a possible 'time' variable was first suggested by Dirac in [18] and is at the base of what is often denoted as the light-front formalism. The latter is today widely used in quantum field theory, and in particular in quantum electrodynamics in the presence of laser pulses; in the latter context it was first introduced in [19]. Its systematic use in classical electrodynamics is less common, though it is often used in studies of radiation reaction (see e.g. [20, 21]), but almost exclusively with EM fields $F^{\mu \nu}$ consisting just of a travelling plane wave $F_{t}^{\mu \nu}(\xi)$; the motion of a classical charged particle in a generic external field of this type has been determined in [4] by solving the Hamilton-Jacobi equation (see section 3.1). A recent exception is Ref. [22], where some interesting superintegrable motions based on symmetric EM fields $F^{\mu \nu}$ not reducing to $F_{t}^{\mu \nu}(\xi)$ are determined. In other works $\xi$ has been adopted ad hoc to simplify the equation of motion of the particle in a particular EM field, e.g. in [23, 24] a monochromatic plane wave and a longitudinal magnetic field (what leads to the phenomenon of cyclotron autoresonance). The main purpose of this paper is therefore a systematic description and development of the lightfront formalism in classical electrodynamics, both in vacuum and in plasmas; a number of significant applications are presented as illustrations of its advantages. Among the latter, also a few new general solutions in closed form in the presence of uniform static EM fields.

The plan of the paper is as follows. In section 2 we first formulate the method for a single charged particle under a general EM field; the Hamiltonian and the Hamilton equations turn out to be rational in the unknowns $\hat{\boldsymbol{x}}, \hat{\boldsymbol{p}}^{\perp}, \hat{s}$. Then we apply it to the case that the EM field is the sum $F=F_{t}+F_{s}$ of a static part and a traveling-wave part (section 2.1) or to the case that the EM potential is independent of the transverse coordinates (section 2.2). In either case we prove several general properties of the solutions; in particular, we show that in the case of section 2.2 integrating the equations of motion reduces to solving a Hamiltonian system with one degree of freedom; this can be done in closed form or numerically (depending on the cases) in the wave-particle interaction region, and by quadrature outside (as there energy is conserved). In section 3 we illustrate the method and these properties while determining the explicit solutions under a general EM wave superposed to various combinations of uniform static fields; these examples are exactly integrable and pedagogical for the issue of extreme 
accelerations. More precisely: we (re)derive in few lines the solutions [4, 25, 26] when the static electric and magnetic fields $\boldsymbol{E}_{s}, \boldsymbol{B}_{s}$ are zero (section 3.1), or have only uniform longitudinal components (one or both: sections 3.2, 3.3, 3.4), or beside the latter have uniform transverse components fulfilling $\boldsymbol{B}_{s}^{\perp}=\mathbf{k} \wedge \boldsymbol{E}_{s}^{\perp}$ (section 3.5); here $\perp$ denotes the component orthogonal to the direction $\mathbf{k}$ of propagation of the pulse. Section 3.1 includes a rigorous statement (Corollary 2) and proof of a generalized version [28] of the socalled Lawson-Woodward no-go theorem [29, 30, 31, 32, 33]; the latter states that the final energy variation of a charged particle induced by an EM pulse is zero under some rather general conditions (motion in vacuum, zero static fields, etc), in spite of the large energy variations during the interaction. To obtain large final energy variations one has thus to violate one of these general conditions. The case treated in section 3.3 yields the known and already mentioned phenomenon of cyclotron autoresonance, which we recall in appendix 5.5; we solve in few lines the equation of motion without the $\beta \simeq 1$ and the monochromaticity assumptions of $[23,24]$, i.e. in a generic plane travelling wave. Whereas we have not found in the literature our general solutions for the cases treated in sections 3.2, 3.4, 3.5. In section 4 we show how to extend our approach to multi-particle systems and plasmas in hydrodynamic conditions. In section 4.1 we specialize it to plane plasma problems; two components of the Maxwell equations can be solved in terms of the other unknowns, and if the plasma is initially in equilibrium we are even able to reduce the system of partial differential equations (PDEs), for short times after the beginning of the interaction with the EM wave, to a family (parametrized - in the Lagrangian description - by the initial position $\boldsymbol{X}$ of the generic electrons fluid element) of decoupled systems of Hamiltonian ODEs with one degree of freedom of the type considered in section 2.2; the latter can be solved numerically. The solutions of section 4.1 can be used to describe the initial motion of the electrons at the interface between the vacuum and a cold low density plasma while a short laser pulse (in the form of a travelling wave) impacts normally onto the plasma. In particular one can derive the socalled slingshot effect $[34,15,16]$, i.e. the backward acceleration and expulsion of high energy electrons just after the laser pulse has hit the surface of the plasma; we illustrate these solutions in the simple case of a step-shaped initial plasma density. Finally, in the appendix we also show (section 5.3) that the change of 'time' $t \mapsto \xi$ induces a generalized canonical (i.e. contact) transformation and determine (section 5.4) rigorous asymptotic expansions in $1 / k$ of definite integrals of the form $\int_{-\infty}^{\xi} d y f(y) e^{i k y}$; the leading term is usually used to approximate slow modulations of monochromatic waves. However we stress that, since Fourier analysis and related notions play no role in the general framework, our method can be applied to all kind of travelling waves, ranging from (almost) monochromatic to so-called "impulses", which contain few, one or even no complete cycle.

\section{Contents}

1 Introduction $\quad 1$

2 General formulation of the single particle dynamics 5

2.1 Dynamics under travelling waves and static fields $\boldsymbol{E}_{s}, \boldsymbol{B}_{s} \ldots \ldots$. . . . 8

2.2 Dynamics under $A^{\mu}$ independent of the transverse coordinates . . . . . . . . 11 
2.2.1 Dynamics under travelling waves and $z$-dependent $\boldsymbol{E}_{s}=\boldsymbol{E}_{s}^{\|} \quad \ldots \ldots$

3 Exact solutions under travelling waves and uniform static fields $\boldsymbol{E}_{s}, \boldsymbol{B}_{s} \quad 13$

$3.1 \boldsymbol{E}_{s}=\boldsymbol{B}_{s}=\mathbf{0}$, and the Lawson-Woodward theorem . . . . . . . . . . . 13

$3.2 \boldsymbol{E}_{s}=\boldsymbol{E}_{s}^{\|}=$const, $\boldsymbol{B}_{s}=\mathbf{0}$ : acceleration, deceleration on a 'slope' . . . . . . 16

$3.3 \boldsymbol{E}_{s}=\mathbf{0}, \boldsymbol{B}_{s}=\boldsymbol{B}_{s}^{\|}=$const, and cyclotron autoresonance . . . . . . . . . 19

3.4 Constant longitudinal $\boldsymbol{E}_{s}=\boldsymbol{E}_{s}^{\|}, \boldsymbol{B}_{s}=\boldsymbol{B}_{s}^{\|} \ldots \ldots \ldots \ldots$

3.5 Adding constant $\boldsymbol{E}_{s}^{\perp}$ and $\boldsymbol{B}_{s}^{\perp}=\mathbf{k} \wedge \boldsymbol{E}_{s}^{\perp}$ to $\boldsymbol{E}_{s}^{\|}, \boldsymbol{B}_{s}^{\|} \ldots \ldots \ldots$

4 Plasmas in the hydrodynamic approximation $\quad 20$

4.1 Plane problems. EM wave hitting a plasma at equilibrium . . . . . . . . . 22

5 Appendix $\quad 27$

5.1 Lagrange equations in the presence of additional forces . . . . . . . . . . 27

5.2 Proof of Proposition 1 and of eq. (31) . . . . . . . . . . . . . . . 28

5.3 Generalized canonical transformations . . . . . . . . . . . . . . . 29

5.4 Estimates of oscillatory integrals . . . . . . . . . . . . . . . 30

5.5 Cyclotron autoresonance . . . . . . . . . . . . . . . 31

\section{General formulation of the single particle dynamics}

By (1b) the particle cannot reach the speed of light, $|\dot{\boldsymbol{x}}|<c$. Given a solution $\boldsymbol{x}(t)$ of (1) let

$$
\xi(t):=c t-z(t), \quad \Rightarrow \quad \dot{\xi}(t)=c-\dot{z}(t)>0 .
$$

The inequality follows from $|\dot{\boldsymbol{x}}|<c$ and implies that we can use the light-like coordinate $\xi=c t-z$ instead of $t$ as the independent (or 'time') variable. In other words, this is possible because the particle worldline intersects every $\xi=$ cost hyperplane in Minkowski spacetime exactly once (see fig. 1 left). If $A_{\mu}(\mathrm{x})$ [we abbreviate $\mathrm{x}=(c t, \boldsymbol{x})$ ] contains a travelling wave part $\alpha_{\mu}(c t-z)$, then $\boldsymbol{E}, \boldsymbol{B}$ in (1) contain terms $\alpha_{\mu}^{\prime}[c t-z(t)]$ which depend on the unknown combination $c t-z(t)$ generally in a highly nonlinear way. If $\left|\alpha_{\mu}^{\prime \prime} \Delta z\right| \ll\left|\alpha_{\mu}^{\prime}\right|$ (nonrelativistic regime) we can simplify the equations approximating $\alpha_{\mu}^{\prime}[c t-z(t)]$ by the known time-dependent force $\alpha_{\mu}^{\prime}\left(c t-z_{0}\right)$, so that the unknown $z(t)$ no more appears in the argument. Otherwise, we can obtain the same result by the change $t \mapsto \xi$, which makes the argument of $\alpha_{\mu}^{\prime}(\xi)$ an independent variable. Let $\hat{\boldsymbol{x}}(\xi)$ be the position as a function of $\xi$, i.e. the position of the intersection (in Minkowski spacetime) of the worldline $\lambda$ with the hyperplane $c t-z=\xi$ (see fig. 1 left); in other words, this function is determined by the condition $\hat{\boldsymbol{x}}[\xi(t)] \equiv \boldsymbol{x}(t)$. More generally we shall put a caret to distinguish the dependence of a dynamical variable on $\xi$ rather than on $t$, e.g. $\hat{\boldsymbol{p}}[\xi(t)] \equiv \boldsymbol{p}(t)$, and $\hat{f}(\xi, \hat{\boldsymbol{x}}):=f[(\xi+\hat{z}) / c, \hat{\boldsymbol{x}}]$ for any given function $f(t, \boldsymbol{x})$. Moreover we shall abbreviate every total derivative with respect to $t, \xi$ by a dot and a prime, respectively. By construction, the variables $\boldsymbol{x}, \boldsymbol{p}, \ldots$ take the values $\hat{\boldsymbol{x}}(\xi), \hat{\boldsymbol{p}}(\xi), \ldots$ at the spacetime point where a value $\alpha_{\mu}(\xi)$ of $\alpha_{\mu}$ reaches the particle; if e.g. $\alpha_{\mu}$ has a maximum at $\bar{\xi}$ then $\hat{\boldsymbol{x}}(\bar{\xi})$ is the value of $\hat{\boldsymbol{x}}$ where (and when) such a maximum $\alpha_{\mu}(\bar{\xi})$ reaches the particle. 


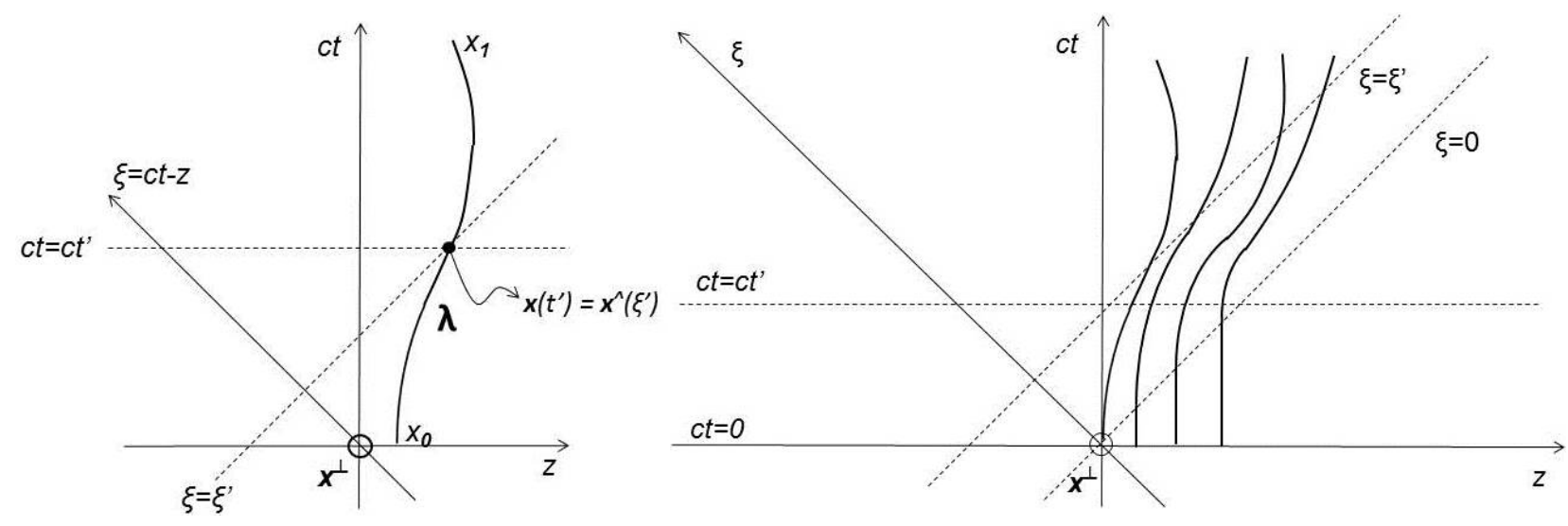

Figure 1: Left: any time-like worldine can be parametrized by the lightlike coordinate $\xi=c t-z$ because it intersects any hyperplane $\xi=$ const exactly once. Right: for a plasma in hydrodynamic conditions no two different fluid elements' worldlines can intersect.

The inverse $\hat{t}(\xi)$ of $\xi(t)$ and its derivative are given by

$$
c \hat{t}(\xi)=\xi+\hat{z}(\xi), \quad c \hat{t}^{\prime}(\xi)=1+\hat{z}^{\prime}(\xi)>0 .
$$

We denote Minkowski spacetime points as $\mathrm{x} \equiv(c t, \boldsymbol{x})$. Given points $\mathrm{x}_{0}, \mathrm{x}_{1}$ with $\mathrm{x}_{1}$ in the causal cone of $\mathrm{x}_{0}$, let $\Lambda$ be the set of time-like curves from $\mathrm{x}_{0}$ to $\mathrm{x}_{1}$. Given a $\lambda \in \Lambda$, we can use in the corresponding action functional of the particle either $t$ or $\xi$ as a parameter on $\lambda$ :

$$
S(\lambda)=-\int_{\lambda} m c^{2} d \tau+q A(\mathrm{x})=-\int_{t_{0}}^{t_{1}} d t \underbrace{\frac{m c^{2}+q u^{\mu} A_{\mu}}{\gamma}}_{L[\boldsymbol{x}, \hat{\boldsymbol{x}}, t]}=-\int_{\xi_{0}}^{\xi_{1}} \frac{d \xi}{c} \underbrace{\frac{m c^{2}+q \hat{u}^{\mu} \hat{A}_{\mu}}{\hat{s}}}_{\mathcal{L}\left[\hat{\boldsymbol{x}}, \hat{\boldsymbol{x}}^{\prime}, \xi\right]} .
$$

Here $A(\mathrm{x})=A_{\mu}(\mathrm{x}) d \mathrm{x}^{\mu}=A^{0}(\mathrm{x}) c d t-\boldsymbol{A}(\mathrm{x}) \cdot d \boldsymbol{x}$ is the EM potential 1-form (the dot is the scalar product in Euclidean $\mathbb{R}^{3}$; we raise and lower greek indices by the Minkowski metric $\eta_{\mu \nu}=\eta^{\mu \nu}$, with $\eta_{00}=1, \eta_{11}=-1$, etc.), $(c d \tau)^{2}=(c d t)^{2}-d \boldsymbol{x}^{2}$ is the square of the infinitesimal Minkowski distance ( $\tau$ is the proper time of the particle), $d t / d \tau=\gamma=1 / \sqrt{1-\boldsymbol{\beta}^{2}}$ (with $\boldsymbol{\beta} \equiv \dot{\boldsymbol{x}} / c)$ is the Lorentz relativistic factor, $u=\left(u^{0}, \boldsymbol{u}\right) \equiv(\gamma, \gamma \boldsymbol{\beta})=\left(\frac{p^{0}}{m c^{2}}, \frac{\mathbf{p}}{m c}\right)$ is the 4 -velocity, i.e. the dimensionless version of the 4-momentum, and

$$
s \equiv \frac{d \xi}{d(c \tau)}=\gamma-u^{z}=u^{-}=\gamma\left(1-\beta^{z}\right)>0
$$

is the light-like component $u^{-}$of $u$, as well as the Doppler factor experienced by the particle, and is positive-definite [the first $=$ in (5) follows from $\left.\gamma=d t / d \tau, p^{z}=m d z / d \tau\right]$. We name $s$ the light-like relativistic factor, or shortly the s-factor. In terms of the "hatted" coordinates and their derivatives ${ }^{4}$

$$
\frac{1}{\hat{s}}=\sqrt{1+2 \hat{z}^{\prime}-\hat{\boldsymbol{x}}^{\perp / 2}} .
$$

\footnotetext{
${ }^{4}$ In fact, $\frac{1}{\hat{s}}=\frac{d \tau}{d t} \frac{d(c t)}{d \xi}=\frac{1}{\gamma} \frac{d(c t)}{d \xi}=\sqrt{1-\left(\frac{d \boldsymbol{x}}{c d t}\right)^{2}} \frac{d(c t)}{d \xi}=\sqrt{\left(\frac{c d t}{d \xi}\right)^{2}-\left(\frac{d \boldsymbol{x}}{d \xi}\right)^{2}} \stackrel{(3)}{=} \sqrt{\left(1+\hat{z}^{\prime}\right)^{2}-\hat{\boldsymbol{x}}^{\prime 2}}=\sqrt{1+2 \hat{z}^{\prime}-\hat{\boldsymbol{x}}^{\perp \prime 2}}$.
} 
Here and throughout the paper $\perp$ stands for the component orthogonal to the direction $\mathbf{k}$ of propagation of the EM wave. The change of Lagrangian $L \mapsto \mathcal{L}$ in (4) amounts to the replacements $\gamma \mapsto s$ and $t \mapsto \xi$ as an independent variable. One easily checks that $\hat{\gamma}, \hat{u}^{z}, \hat{\boldsymbol{\beta}}, \hat{\boldsymbol{x}}^{\prime}$ can be expressed as the following rational functions of $\hat{\boldsymbol{u}}^{\perp}, \hat{s}$,

$$
\begin{array}{rr}
\hat{\gamma}=\frac{1+\hat{\boldsymbol{u}}^{\perp 2}+\hat{s}^{2}}{2 \hat{s}}, \quad \hat{u}^{z}=\hat{\gamma}-\hat{s}, \quad \hat{\boldsymbol{\beta}}=\frac{\hat{\boldsymbol{u}}}{\hat{\gamma}}, \\
\hat{\boldsymbol{x}}^{\perp \prime}=\frac{\hat{\boldsymbol{u}}^{\perp}}{\hat{s}}, \quad \hat{z}^{\prime}=\frac{1+\hat{\boldsymbol{u}}^{\perp 2}}{2 \hat{s}^{2}}-\frac{1}{2}
\end{array}
$$

(the first three relations hold also without the caret), i.e. square roots no longer appear in these purely kinematical relations. In the nonrelativistic regime $s \simeq 1$; whereas $\pm u^{z} \gg$ $\sqrt{1+\boldsymbol{u}^{\perp 2}}$ respectively imply $s \ll 1, s \gg 1$. More explicitly $L, \mathcal{L}$ read

$$
\begin{aligned}
L[\boldsymbol{x}, \dot{\boldsymbol{x}}, t] & =-m c^{2} \sqrt{1-\frac{\dot{\boldsymbol{x}}^{2}}{c^{2}}}-q\left[A^{0}(\mathrm{x})-\boldsymbol{A}(\mathrm{x}) \cdot \frac{\dot{\boldsymbol{x}}}{c}\right], \\
\mathcal{L}\left[\hat{\boldsymbol{x}}, \hat{\boldsymbol{x}}^{\prime}, \xi\right] & =\left(1+\hat{z}^{\prime}\right) L\left[\hat{\boldsymbol{x}}, \frac{c \hat{\boldsymbol{x}}^{\prime}}{1+\hat{z}^{\prime}}, \frac{\xi+\hat{z}}{c}\right] \\
& =-m c^{2} \sqrt{1+2 \hat{z}^{\prime}-\hat{\boldsymbol{x}}^{\perp \prime 2}}-q\left(1+\hat{z}^{\prime}\right) \hat{A}^{0}+q \hat{\boldsymbol{x}}^{\prime} \cdot \hat{\boldsymbol{A}}
\end{aligned}
$$

where we have used (3b). By Hamilton's principle, any extremum $\lambda \in \Lambda$ of $S$ is the worldline of a possible motion of the particle with initial position $\boldsymbol{x}_{0}$ at time $t_{0}$ and final position $\boldsymbol{x}_{1}$ at time $t_{1}$; hence it fulfills both Euler-Lagrange equations

$$
\text { (1) } \Leftrightarrow \frac{d}{d t} \frac{\partial L}{\partial \dot{\boldsymbol{x}}}-\frac{\partial L}{\partial \boldsymbol{x}}=0 \quad \Leftrightarrow \quad \frac{d}{d \xi} \frac{\partial \mathcal{L}}{\partial \hat{\boldsymbol{x}}^{\prime}}-\frac{\partial \mathcal{L}}{\partial \hat{\boldsymbol{x}}}=0 .
$$

Having solved (11c), we obtain the solution of (11) by setting $\boldsymbol{x}(t)=\hat{\boldsymbol{x}}[\xi(t)]$, where $\xi(t)$ is obtained inverting (3a).

Incidentally, the presence of additional forces (beside the electromagnetic ones) can be incorporated in the formalism modifying all right-hand sides of (11), see Appendix 5.1.

We can rephrase (11c) in Hamiltonian form. The derivatives appearing in (11c) read

$$
\begin{aligned}
& \frac{\partial \mathcal{L}}{\partial \hat{\boldsymbol{x}}}=-\left(1+\hat{z}^{\prime}\right) q \frac{\partial \hat{A}^{0}}{\partial \hat{\boldsymbol{x}}}+q \frac{\partial \hat{A}^{i}}{\partial \hat{\boldsymbol{x}}} \cdot \hat{x}^{i \prime}, \\
& \frac{\partial \mathcal{L}}{\partial \hat{\boldsymbol{x}}^{\perp \prime}}=m c^{2} \hat{\boldsymbol{u}}^{\perp}+q \hat{\boldsymbol{A}}^{\perp}, \\
& \frac{\partial \mathcal{L}}{\partial \hat{z}^{\prime}}=-m c^{2} \hat{s}-q\left(\hat{A}^{0}-\hat{A}^{z}\right) .
\end{aligned}
$$

The Legendre transform gives the Hamiltonian $\hat{H}:=\hat{\boldsymbol{x}}^{\prime} \cdot \partial \mathcal{L} / \partial \hat{\boldsymbol{x}}^{\prime}-\mathcal{L}=\hat{\gamma} m c^{2}+q \hat{A}^{0} ;$ expressing this as functions of $\hat{\boldsymbol{x}}, \hat{\boldsymbol{\Pi}}:=\partial \mathcal{L} / \partial \hat{\boldsymbol{x}}^{\prime}$ we obtain

$$
\hat{H}(\hat{\boldsymbol{x}}, \hat{\boldsymbol{\Pi}} ; \xi)=m c^{2} \frac{1+\hat{s}^{2}+\hat{\boldsymbol{u}}^{\perp 2}}{2 \hat{s}}+q \hat{A}^{0}(\xi, \hat{\boldsymbol{x}}), \quad \text { where }\left\{\begin{array}{l}
\hat{\boldsymbol{u}}^{\perp}=\frac{\hat{\boldsymbol{\Pi}}^{\perp}-q \hat{\boldsymbol{A}}^{\perp}(\xi, \hat{\boldsymbol{x}})}{m c^{2}} \\
\hat{s}=-\frac{\hat{\Pi}^{z}+q\left[\hat{A}^{0}-\hat{A}^{z}\right](\xi, \hat{\boldsymbol{x}})}{m c^{2}}
\end{array}\right.
$$


and we find as usual that the Lagrange equations (11c) are equivalent to the Hamilton ones

$$
\hat{\boldsymbol{x}}^{\prime}=\frac{\partial \hat{H}}{\partial \hat{\boldsymbol{\Pi}}}, \quad \hat{\boldsymbol{\Pi}}^{\prime}=-\frac{\partial \hat{H}}{\partial \hat{\boldsymbol{x}}} .
$$

Remarks 2. Note that, while the usual Hamiltonian $H(\boldsymbol{x}, \boldsymbol{P}, t)=\sqrt{m^{2} c^{4}+(c \boldsymbol{P}-q \boldsymbol{A})^{2}}+q A^{0}$ is the square root of a polynomial in the generalized momenta $\boldsymbol{P}=\partial L / \partial \dot{\boldsymbol{x}}=\boldsymbol{p}+q \boldsymbol{A} / c, \hat{H}$ is a rational function of $\hat{\boldsymbol{\Pi}}$ or, equivalently, of $\hat{\boldsymbol{s}}, \hat{\boldsymbol{u}}^{\perp}$. In (12) the caret over $H$ is justified because $\hat{H}$ coincides with $H(\boldsymbol{x}, \boldsymbol{P}, t)$ when $A^{\mu}, \boldsymbol{x}, \boldsymbol{P}$ are expressed as functions of $\xi$; hence, along the solutions of (13) $\hat{H}$ gives the particle energy expressed as a function of $\xi$. In appendix 5.3 we show that the map $(\boldsymbol{x}, \boldsymbol{P}, t) \mapsto(\boldsymbol{x}, \boldsymbol{\Pi} / c, \xi / c)$ is a generalized canonical (i.e., contact) transformation. In general the new equations can be obtained from the old ones by putting a caret on all dynamical variables and replacing $d / d t \mapsto(c \hat{s} / \hat{\gamma}) d / d \xi$.

In appendix 5.2 we prove

Proposition 1 Eq. (1), or equivalently the Hamilton equations (13), amount to (8) and

$$
\begin{aligned}
& \hat{\boldsymbol{u}}^{\perp \prime}=\frac{q}{m c^{2} \hat{s}}[\hat{\gamma} \hat{\boldsymbol{E}}+\hat{\boldsymbol{u}} \wedge \hat{\boldsymbol{B}}]^{\perp} \\
& \hat{s}^{\prime}=\frac{q}{m c^{2}}\left[\frac{\hat{\boldsymbol{u}}^{\perp}}{\hat{s}} \cdot \hat{\boldsymbol{E}}^{\perp}-\hat{E}^{z}-\frac{\left(\hat{\boldsymbol{u}}^{\perp} \wedge \hat{\boldsymbol{B}}^{\perp}\right)^{z}}{\hat{s}}\right]
\end{aligned}
$$

with $\hat{\gamma}$ as given in (7). Along their solutions

$$
\frac{d \hat{H}}{d \xi}=\frac{\partial \hat{H}}{\partial \xi}
$$

We define the energy gain of the particle in the interval $\left[\xi_{0}, \xi_{1}\right]$ as $\mathcal{E}:=\left[\hat{H}\left(\xi_{1}\right)-\hat{H}\left(\xi_{0}\right)\right] / m c^{2}$ (we have normalized it so that it is dimensionless).

Corollary $1 \hat{H}$ is conserved in a spacetime region where $A^{\mu}$ is independent of $t$. More generally, if $A^{0}, A^{z}$ are independent of then the dimensionless energy gain is given by

$$
\mathcal{E}=\int_{\xi_{0}}^{\xi_{1}} \frac{d \xi}{2 \hat{s}(\xi)} \frac{\partial \hat{v}}{\partial \xi}[\hat{\boldsymbol{x}}(\xi), \hat{\boldsymbol{\Pi}}(\xi) ; \xi], \quad \hat{v}:=\hat{\boldsymbol{u}}^{\perp 2}
$$

Proof: in the first case $\hat{A}^{\mu}$ has no direct dependence on $\xi$, hence $\partial \hat{H} / \partial \xi=0$; in the second $\hat{H}$ depends directly on $\xi$ only through $\hat{v}$, hence $\partial \hat{H} / \partial \xi=(\partial \hat{v} / \partial \xi) / 2 \hat{s}(\xi)$, and the claim follows.

\subsection{Dynamics under travelling waves and static fields $\boldsymbol{E}_{s}, \boldsymbol{B}_{s}$}

We are especially interested in problems in which the EM field is the sum of a transverse travelling wave (the 'pump') and a purely $\boldsymbol{x}$-dependent (i.e. static) part:

$$
\boldsymbol{E}(\mathrm{x})=\boldsymbol{\epsilon}^{\perp}(c t-z)+\boldsymbol{E}_{s}(\boldsymbol{x}), \quad \boldsymbol{B}(\mathrm{x})=\mathbf{k} \wedge \boldsymbol{\epsilon}^{\perp}(c t-z)+\boldsymbol{B}_{s}(\boldsymbol{x}) .
$$


This can be obtained adopting an electromagnetic potential of the same form:

$$
A^{\mu}(\mathrm{x})=\alpha^{\mu}(c t-z)+A_{s}^{\mu}(\boldsymbol{x}) \quad \Leftrightarrow \quad \hat{A}^{\mu}(\xi, \hat{\boldsymbol{x}})=\alpha^{\mu}(\xi)+A_{s}^{\mu}(\hat{\boldsymbol{x}}) .
$$

Choosing the Landau gauges $\left(\partial_{\mu} A^{\mu}=0\right)$ implies that $\boldsymbol{A}_{s}$ must fufill the Coulomb gauges $\left(\nabla \cdot \boldsymbol{A}_{s}=0\right)$, and it must be $\alpha^{z \prime}=\alpha^{0 \prime}, \boldsymbol{\epsilon}^{\perp}=-\boldsymbol{\alpha}^{\perp \prime}, \boldsymbol{E}_{s}=-\nabla A_{s}^{0}, \boldsymbol{B}_{s}=\nabla \wedge \boldsymbol{A}_{s}$. We shall set $\alpha^{z}=\alpha^{0}=0$, as they appear neither in the observables $\boldsymbol{E}, \boldsymbol{B}$ nor in the equations of motion. If we assume that the pump $\boldsymbol{\epsilon}^{\perp}(\xi)$ is continuous (at least piecewise) and

$$
\begin{array}{lll}
\text { either } & \text { a) } & \boldsymbol{\epsilon}^{\perp} \text { has a compact support, } \\
\text { or } & \left.\mathbf{a}^{\prime}\right) & \boldsymbol{\epsilon}^{\perp} \in L^{1}(\mathbb{R}),
\end{array}
$$

we can choose the (unique) $\boldsymbol{\alpha}^{\perp}(\xi)$ going to zero as $\xi \rightarrow-\infty$ :

$$
\boldsymbol{\alpha}^{\perp}(\xi)=-\int_{-\infty}^{\xi} d \xi^{\prime} \boldsymbol{\epsilon}^{\perp}\left(\xi^{\prime}\right) ;
$$

note that if $\boldsymbol{\epsilon}^{\perp}(\xi)$ vanishes for $\xi \leq \xi_{0}$, so does $\boldsymbol{\alpha}^{\perp}(\xi)$. The so defined $\boldsymbol{\alpha}^{\perp}$ is a physical observable (the gauge freedom has been completely fixed). Condition (19a) implies $\boldsymbol{E}_{=} \boldsymbol{E}_{s}$ outside the strip $0 \leq c t-z \leq l$, if the interval $[0, l]$ contains the support of $\boldsymbol{\epsilon}^{\perp}$; (19a') implies $\boldsymbol{E} \rightarrow \boldsymbol{E}_{s}$ as $z \rightarrow \infty$ at every fixed $t$, or equivalently as $t \rightarrow-\infty$ at every fixed $\boldsymbol{x}$.

The present approach allows to treat on the same footing all such $\boldsymbol{\epsilon}^{\perp}$, namely very different travelling waves, regardless of their Fourier analysis. In particular:

1. A modulated monochromatic wave

$$
\boldsymbol{\epsilon}^{\perp}(\xi)=\epsilon(\xi) \boldsymbol{\epsilon}_{o}^{\perp}(\xi), \quad \boldsymbol{\epsilon}_{o}^{\perp}(\xi)=\mathbf{i} a_{1} \cos \left(k \xi+\varphi_{1}\right)+\mathbf{j} a_{2} \sin \left(k \xi+\varphi_{2}\right)
$$

with some wave number $k$ (not to be confused with the unit vector $\mathbf{k}$ in the direction of propagation of the wave, in boldface!), modulating amplitude $\epsilon(\xi) \geq 0$ fulfilling (19) and elliptic polarization determined by some $a_{h}, \varphi_{h} \in \mathbb{R}$ (with $a_{1}^{2}+a_{2}^{2}=1$ ). Let $\boldsymbol{\epsilon}_{p}^{\perp}:=-\boldsymbol{\epsilon}_{o}^{\perp \prime} / k$. In particular we shall consider

$$
\begin{aligned}
& \boldsymbol{\epsilon}_{o}^{\perp}(\xi)=\mathbf{i} \cos k \xi, \quad \boldsymbol{\epsilon}_{p}^{\perp}(\xi)=\mathbf{i} \sin k \xi \quad \text { (linearly polarized), or } \\
& \boldsymbol{\epsilon}_{o}^{\perp}(\xi)=\mathbf{i} \cos k \xi+\mathbf{j} \sin k \xi, \quad \boldsymbol{\epsilon}_{p}^{\perp}(\xi)=\mathbf{i} \sin k \xi-\mathbf{j} \cos k \xi \quad \text { (circularly polarized). }
\end{aligned}
$$

In appendix 5.4 we show that under rather general assumptions

$$
\boldsymbol{\alpha}^{\perp}(\xi)=-\frac{\epsilon(\xi)}{k} \boldsymbol{\epsilon}_{p}^{\perp}(\xi)+O\left(\frac{1}{k^{2}}\right) \simeq-\frac{\epsilon(\xi)}{k} \boldsymbol{\epsilon}_{p}^{\perp}(\xi)
$$

giving upper bounds for the involved remainder $O\left(1 / k^{2}\right)$. For slow modulations (i.e. $\left|\epsilon^{\prime}\right| \ll|k \epsilon|$ almost everywhere on the support) - like the ones characterizing most conventional applications, like radio broadcasting, ordinary laser pulses, etc. - the right estimate is very good. Consequently, if $\epsilon(\xi)$ goes to zero also as $\xi \rightarrow \infty$, then $\boldsymbol{\alpha}^{\perp}(\xi), \hat{v}(\xi)$ approximately do as well. Given a modulating amplitude $\epsilon_{0}(\xi)$ of such a type, consider the rescaled one

$$
\epsilon(\xi ; \eta):=\epsilon_{0}(\xi / \eta)
$$

in the scaling limit $\eta \rightarrow \infty$ the $\simeq$ in (23) becomes a strict equality and $\boldsymbol{\epsilon}^{\perp}$ becomes monochromatic. 
2. A superposition of several waves of the previous kind.

3. At the antipodes, a wave with very few cycles [35], or even an 'impulse' [36, 37, 38, 8], i.e. a wave with one, a 'fraction' of a cycle (such waves are emitted e.g. during transients, like electric discharges, or can be manufactured [8] even with high intensity and frequency).

Proposition 2 In an EM field (17) the equations of motion (13) amount to (8) and

$$
\begin{aligned}
& \hat{\boldsymbol{u}}^{\perp \prime}=\frac{q}{m c^{2}}\left[\left(1+\hat{z}^{\prime}\right) \hat{\boldsymbol{E}}_{s}^{\perp}+\left(\hat{\boldsymbol{x}}^{\prime} \wedge \hat{\boldsymbol{B}}_{s}\right)^{\perp}+\boldsymbol{\epsilon}^{\perp}(\xi)\right], \\
& \hat{s}^{\prime}=\frac{-q}{m c^{2}}\left[\hat{E}_{s}^{z}-\hat{\boldsymbol{x}}^{\perp \prime} \cdot \hat{\boldsymbol{E}}_{s}^{\perp}+\left(\hat{\boldsymbol{x}}^{\perp \prime} \wedge \hat{\boldsymbol{B}}_{s}^{\perp}\right)^{z}\right] ;
\end{aligned}
$$

on a solution the energy gain (16) is obtained integrating the expression

$$
\hat{H}^{\prime}=\frac{m c^{2}}{2 \hat{s}} \frac{\partial \hat{v}}{\partial \xi}=\frac{\hat{\boldsymbol{u}}^{\perp}}{\hat{s}} \cdot q \boldsymbol{\epsilon}^{\perp}=q \hat{\boldsymbol{x}}^{\perp \prime} \cdot \boldsymbol{\epsilon}^{\perp}
$$

The proof is a straightforward computation. As evident, the argument of the rapidly varying function $\boldsymbol{\epsilon}^{\perp}$ does no longer contain the unknown $z(t)$, but is the independent variable $\xi$. In particular, if $\boldsymbol{E}_{s}, \boldsymbol{B}_{s}=$ const then eq. (25) are immediately integrated to yield

$$
\begin{aligned}
& \hat{\boldsymbol{u}}^{\perp}=\frac{q}{m c^{2}}\left[\boldsymbol{K}^{\perp}-\boldsymbol{\alpha}^{\perp}(\xi)+(\xi+\hat{z}) \boldsymbol{E}_{s}^{\perp}+\left(\hat{\boldsymbol{x}} \wedge \boldsymbol{B}_{s}\right)^{\perp}\right], \\
& \hat{s}=\frac{-q}{m c^{2}}\left[K^{z}+\xi E_{s}^{z}-\hat{\boldsymbol{x}}^{\perp} \cdot \boldsymbol{E}_{s}^{\perp}+\left(\hat{\boldsymbol{x}}^{\perp} \wedge \boldsymbol{B}_{s}\right)^{z}\right]
\end{aligned}
$$

(the integration constants $K^{j}$ are fixed by the initial conditions), or more explictly

$$
\begin{aligned}
& \hat{u}^{x}=w^{x}(\xi)+\left(e^{x}-b^{y}\right) \hat{z}+b \hat{y}, \\
& \hat{u}^{y}=w^{y}(\xi)+\left(e^{y}+b^{x}\right) \hat{z}-b \hat{x}, \\
& \hat{s}=w^{z}(\xi)+\left(e^{x}-b^{y}\right) \hat{x}+\left(e^{y}+b^{x}\right) \hat{y}
\end{aligned}
$$

[here we have introduced the dimensionless functions $\boldsymbol{w}^{\perp}(\xi):=q\left[\boldsymbol{K}^{\perp}-\boldsymbol{\alpha}^{\perp}(\xi)+\xi \boldsymbol{E}_{s}^{\perp}\right] / m c^{2}$, $w^{z}(\xi):=-q\left(K^{z}+\xi E_{s}^{z}\right) / m c^{2}$ and the constants $\left.\boldsymbol{e}^{\perp}:=q \boldsymbol{E}_{s}^{\perp} / m c^{2}, \boldsymbol{b}^{\perp}+b \mathbf{k}:=q \boldsymbol{B}_{s} / m c^{2}\right]$. Hence,

Proposition 3 If the EM field is of the form (17), then solving the Hamilton equations (13) amounts to solving the system of three first order ODEs in rational form in the unknowns $\hat{x}, \hat{y}, \hat{z}$ which is obtained replacing (28) in (8).

To start illustrating the advantages of the present approach let us compare these equations with the usual Hamilton equations $\dot{\boldsymbol{x}}=\partial H / \partial \boldsymbol{P}, \dot{\boldsymbol{P}}=-\partial H / \partial \boldsymbol{x}$. The former amount to $\dot{\boldsymbol{x}}=\boldsymbol{u} / \sqrt{1+\boldsymbol{u}^{2}}$, which have no rational form, and

$$
\dot{\boldsymbol{u}}(t)=\frac{q}{m c}\left\{\boldsymbol{E}_{s}+\left(\frac{\dot{\boldsymbol{x}}}{c} \wedge \boldsymbol{B}_{s}\right)+\left(\frac{\dot{\boldsymbol{x}}^{\perp}}{c} \cdot \boldsymbol{\epsilon}^{\perp}[c t-z(t)]\right) \mathbf{k}-\frac{1}{c} \frac{d}{d t} \boldsymbol{\alpha}^{\perp}[c t-z(t)]\right\} .
$$


Contrary to (25), the unknown $c t-z(t)$ appears in the argument of the rapidly varying function $\boldsymbol{\epsilon}^{\perp}, \boldsymbol{\alpha}^{\perp}$. Moreover, if $\boldsymbol{E}_{s}, \boldsymbol{B}_{s}=$ const then, although the transverse components of eq. (29) are also immediately integrated to yield a relation equivalent to (27a)

$$
\boldsymbol{u}^{\perp}=\frac{q}{m c^{2}}\left\{\boldsymbol{K}^{\perp}-\boldsymbol{\alpha}^{\perp}[c t-z(t)]+c t \boldsymbol{E}_{s}^{\perp}+\left(\boldsymbol{x} \wedge \boldsymbol{B}_{s}\right)^{\perp}\right\}
$$

the right-hand side is nonlinear in the unknown $z(t)$ [while the right-hand side of (27a) is linear in the unknown $\hat{\boldsymbol{x}}(\xi)$ ], and the longitudinal component of eq. (29) is not integrated in any trivial and general way. Also the determination of the energy gain as a function of $t$ is quite more complicated.

\subsection{Dynamics under $A^{\mu}$ independent of the transverse coordinates}

Further advantages of our approach can be disclosed also whenever the gauge potential is independent of $\boldsymbol{x}^{\perp}, A^{\mu}=A^{\mu}(t, z)$. Then $\partial \hat{H} / \partial \hat{\boldsymbol{x}}^{\perp}=0$, and the transverse component of (14b) implies $q \boldsymbol{K}^{\perp} \equiv \hat{\boldsymbol{\Pi}}^{\perp}=$ const, i.e. the known result

$$
\hat{\boldsymbol{u}}^{\perp}=\frac{q}{m c^{2}}\left[\boldsymbol{K}^{\perp}-\hat{\boldsymbol{A}}^{\perp}(\xi, \hat{z})\right] ;
$$

this expresses $\hat{\boldsymbol{u}}^{\perp}$ in terms of $\hat{\boldsymbol{A}}^{\perp}(\xi, \hat{z})$ and $\boldsymbol{K}^{\perp}$, which is determined by the initial conditions ${ }^{5}$. Eq. (30) applies in particular when $\boldsymbol{E}, \boldsymbol{B}$ are of the form (17) with $\boldsymbol{E}_{s}=\mathbf{k} E_{s}^{z}(z)$ (longitudinal field), $\boldsymbol{B}_{s}=\boldsymbol{B}_{s}^{\perp}(z)$ [we can choose the static part (18) of the gauge potential independent of $\boldsymbol{x}^{\perp}$ as well, $A_{s}^{\mu}=A_{s}^{\mu}(z)$ ]. Replacing (30) in the longitudinal component of (13) we obtain (see appendix 5.2)

$$
\hat{z}^{\prime}=\frac{1+\hat{v}}{2 \hat{s}^{2}}-\frac{1}{2}, \quad \quad m c^{2} \hat{s}^{\prime}=-q E_{s}^{z}(\hat{z})-\frac{m c^{2}}{2 \hat{s}} \frac{\partial \hat{v}}{\partial \hat{z}} .
$$

This is a system of two first order ODEs in the unknowns $\hat{z}(\xi), \hat{s}(\xi)$. Having solved (31), expressing $\hat{\boldsymbol{u}}, \gamma$ in terms of $\hat{s}(\xi), \hat{z}(\xi), \hat{\boldsymbol{A}}^{\perp}[\xi, \hat{z}(\xi)]$ through (7), (30), and integrating over $\xi$, one determines in closed form also $\hat{t}(\xi), \hat{\boldsymbol{x}}^{\perp}(\xi)$, and thus the whole $\hat{\boldsymbol{x}}(\xi)$ :

$$
\begin{array}{cc}
\hat{\boldsymbol{x}}(\xi)=\boldsymbol{x}_{0}+\hat{\boldsymbol{Y}}(\xi), & \text { where } \hat{\boldsymbol{Y}}(\xi) \equiv \int_{\xi_{0}}^{\xi} d \zeta \frac{\hat{\boldsymbol{u}}(\zeta)}{\hat{s}(\zeta)}, \\
c \hat{t}(\xi)=\xi+\hat{z}(\xi)=c t_{0}+\hat{\Xi}(\xi), & \text { where } \quad \hat{\Xi}(\xi) \equiv \int_{\xi_{0}}^{\xi} d \zeta \frac{\hat{\gamma}(\zeta)}{\hat{s}(\zeta)}=\xi-\xi_{0}+\hat{Y}^{z}(\xi) .
\end{array}
$$

Clearly $\hat{\Xi}(\xi)$ is strictly increasing. Inverting (33) we find $\xi(t)=\hat{\Xi}^{-1}\left(c t-c t_{0}\right)$ and setting $\boldsymbol{x}(t)=\hat{\boldsymbol{x}}[\xi(t)]$ we finally obtain the original unknown:

$$
\boldsymbol{x}(t)=\boldsymbol{x}_{0}+\hat{\boldsymbol{Y}}\left[\hat{\Xi}^{-1}\left(c t-c t_{0}\right)\right] .
$$

Summarizing, we have shown

\footnotetext{
${ }^{5}$ Under the above assumptions $\boldsymbol{A}_{s}^{\perp}$ is recovered from $\boldsymbol{B}_{s}^{\perp}$ through $\boldsymbol{A}_{s}^{\perp}(z)=\int_{z_{0}}^{z} d z^{\prime} \boldsymbol{B}_{s}^{\perp}\left(z^{\prime}\right) \wedge \mathbf{k}+\boldsymbol{a}^{\perp}$, so it is determined up to the additive constant $\boldsymbol{a}^{\perp}$ (residual gauge freedom). $\left(\boldsymbol{K}^{\perp}-\boldsymbol{a}^{\perp}\right) q / m c^{2}$ is determined by the initial conditions, so that the physical observable $\hat{\boldsymbol{u}}^{\perp}$ is independent of the choice of $\boldsymbol{a}^{\perp}$, as it must be. Similarly, $A_{s}^{0}(z)=-\int_{z_{0}}^{z} d z^{\prime} E_{s}^{z}\left(z^{\prime}\right)+$ const, whereas in the Coulomb gauge $A_{s}^{z}$ can be chosen as zero.
} 
Proposition 4 If $A_{\mu}$ are independent of $\boldsymbol{x}^{\perp}$ the resolution of the equations of motion is reduced to solving the 1-dimensional system (31). The other unknowns are then obtained from formulae (30), (32-34).

\subsubsection{Dynamics under travelling waves and $z$-dependent $\boldsymbol{E}_{s}=\boldsymbol{E}_{s}^{\|}$}

If in addition $\boldsymbol{B}_{s} \equiv 0$, then in (18) we can assume $\boldsymbol{A}_{s} \equiv 0$ without loss of generality (by the Coulomb gauge). In the notation introduced after (28), eq. (30) becomes $\hat{\boldsymbol{u}}^{\perp}(\xi)=\boldsymbol{w}^{\perp}(\xi)$ and $\hat{v}=\hat{\boldsymbol{u}}^{\perp 2}$, i.e. they are already known. Equations (31), (12), (15) reduce to

$$
\begin{aligned}
& \hat{z}^{\prime}=\frac{1+\hat{v}}{2 \hat{s}^{2}}-\frac{1}{2}, \quad m c^{2} \hat{s}^{\prime}=-q E_{s}^{z}(\hat{z}), \\
& \hat{H}(\hat{z}, \hat{s} ; \xi)=m c^{2} \frac{1+\hat{s}^{2}+\hat{v}(\xi)}{2 \hat{s}}+q A_{s}^{0}(\hat{z}), \quad \hat{H}^{\prime}=\frac{\hat{v}^{\prime}}{\hat{s}},
\end{aligned}
$$

Since $\left(\hat{z}, \hat{\Pi}^{z} / c\right) \mapsto(\hat{z},-m c \hat{s})$ is a canonical transformation, here we can adopt also $(\hat{z},-m c \hat{s})$ as canonical coordinates. It is now straightforward to prove the following

\section{Remarks 2.2.1 (General properties of the solutions):}

1. In a region where $\boldsymbol{\epsilon}^{\perp}(\xi)=0$ then $\hat{v}(\xi)=v_{c}=$ const and:

(a) $\hat{H}$ is conserved, the solution $(\hat{z}(\xi), \hat{s}(\xi))$ moves along the corresponding energy level curve $C_{E}$ of equation $\hat{H}(\hat{s}, \hat{z})=E$ and can be determined by quadrature.

(b) If the electrostatic potential energy $U(z) \equiv q A_{s}^{0}(z)$ is bounded from below, then there exist $s_{m}, s_{M}$ such that $0<s_{m} \leq s(\xi) \leq s_{M}$.

(c) If $U(z)$ has a minimum $U_{0}$ at some $z=z_{0}$, then for sufficiently low $E>U_{0}+$ $m c^{2} \sqrt{1+v_{c}}$ all $C_{E}$ are cycles around $(\hat{z}, \hat{s})=\left(z_{0}, \sqrt{1+v_{c}}\right)$ (longitudinal oscillations).

2. The maximal domain of any solution is of the type $\xi \in]-\infty, \xi_{f}[$. If $U(z)$ is bounded from below as $z \rightarrow \infty$, what is always the case in reality, then $\xi_{f}=\infty$ and $t_{f}=$ $\left[\xi_{f}+\hat{z}\left(\xi_{f}\right)\right] / c=\infty$. Furthermore, even if we allow $U$ such that $U(z) \rightarrow-\infty$ as $z \rightarrow \infty$ (this is convenient if e.g. we wish to study the particle motion in a region $V$ where $q E_{s}^{z}$ is a positive constant, without specifying how large $V$ is), then $\xi_{f}$ may be finite and $(\hat{z}(\xi), \hat{s}(\xi)) \rightarrow(\infty, 0)$ as $\xi \rightarrow \xi_{f}$ [see e.g. eq. (42)]; but also in such cases $t_{f}=$ $\left[\xi_{f}+\hat{z}\left(\xi_{f}\right)\right] / c=\infty$, and the solution $(z(t), s(t))$ is defined for all $t \in \mathbb{R}$, as expected.

3. The final transverse momentum is $m c \boldsymbol{u}_{f}^{\perp}$, where $\boldsymbol{u}_{f}^{\perp}:=\hat{\boldsymbol{u}}^{\perp}\left(\xi_{f}\right)=\boldsymbol{w}^{\perp}\left(\xi_{f}\right)$. If $\boldsymbol{\epsilon}^{\perp}$ is slowly modulated and $\boldsymbol{\epsilon}^{\perp}\left(\xi_{f}\right)=0$, then $\boldsymbol{u}_{f}^{\perp} \simeq q \boldsymbol{K}^{\perp} / m c^{2}$; in particular, if $\boldsymbol{u}^{\perp}=0$ before the wave-particle interaction, then $\boldsymbol{K}^{\perp}=0$ and $\boldsymbol{u}_{f}^{\perp} \simeq 0$ as well (cf. appendix 5.4), i.e. the final transverse momentum and velocity approximately vanish.

4. The energy gain (16) becomes

$$
\mathcal{E}=\int_{\xi_{0}}^{\xi_{1}} d \xi \frac{\hat{v}^{\prime}(\xi)}{2 \hat{s}(\xi)} A c t a=\int_{\xi_{0}}^{\xi_{1}} d \xi \frac{\hat{v}(\xi) \hat{s}^{\prime}(\xi)}{2 \hat{s}^{2}(\xi)}+\frac{\hat{v}\left(\xi_{1}\right)}{2 \hat{s}\left(\xi_{1}\right)}-\frac{\hat{v}\left(\xi_{0}\right)}{2 \hat{s}\left(\xi_{0}\right)} .
$$


In the last expression: the last term vanishes if $\xi_{0} \leq 0, \xi_{0}=-\infty$, respectively (resp.) in the cases (19a), (19a'); by 3., also the second term can be neglected in the case of a slowly modulated wave $(21)$ with $\epsilon\left(\xi_{1}\right)=0$. Then, since $\hat{v} / \hat{s}^{2}$ is positive definite, the energy gain will be automatically positive (resp. negative) if $\hat{s}(\xi)$ is growing (resp. decreasing) for all $\xi_{0}<\xi<\xi_{1}$. Correspondingly, the interaction with the pump can be

used to accelerate (resp. decelerate) the particle. Choosing $\xi_{1}=\xi_{f}$ the last two terms in (37) vanish and we obtain the final energy gain $\mathcal{E}_{f}$ across the whole wave-particle interaction.

5. $\hat{s}(\xi)$ is least sensitive to fast oscillations of the 'pump' $\boldsymbol{\epsilon}^{\perp}$ : from (35) it follows

$$
\hat{z}(\xi)=z_{0}+\int_{\xi_{0}}^{\xi} d \zeta \frac{\hat{v}(\zeta)}{2 \hat{s}^{2}(\zeta)}+\int_{\xi_{0}}^{\xi} d \zeta\left[\frac{1}{2 \hat{s}^{2}(\zeta)}-\frac{1}{2}\right], \quad \hat{s}(\xi)=s_{0}-\int_{\xi_{0}}^{\xi} d \zeta \frac{q E_{s}^{z}[\hat{z}(\zeta)]}{m c^{2}}
$$

The fast oscillations of $\hat{v}$ [e.g. $\hat{v}(\xi) \sim(1-\cos 2 k \xi) \epsilon^{2}(\xi) / 2$ if $\boldsymbol{\epsilon}^{\perp}$ is a slowly modulated, linearly polarized wave (21-22) and $\boldsymbol{K}^{\perp}=0$ ] induce by the first integration much smaller relative oscillations of $\hat{z}$, because $\hat{v} / \hat{s}^{2} \geq 0$ and its integral is a growing function of $\xi$; the last integration averages the residual small oscillations of $E_{s}^{z}[\hat{z}(\xi)]$ to yield an essentially smooth $\hat{s}(\xi)$. The functions $\hat{\gamma}(\xi), \hat{\boldsymbol{\beta}}(\xi), \hat{\boldsymbol{u}}(\xi), \ldots$, which are recovered through (7), obviously do not share the same remarkable property, nor do $\gamma(t), \boldsymbol{\beta}(t), \boldsymbol{u}(t), \ldots$ See the graphs of the examples treated below.

These general properties play a role e.g. in the cases considered in sections $3.2,4.1$.

\section{Exact solutions under travelling waves and uniform static fields $\boldsymbol{E}_{s}, \boldsymbol{B}_{s}$}

In this section we illustrate the power of our approach solving the equations: i) when $\boldsymbol{E}_{s}=$ $\boldsymbol{B}_{s}=\mathbf{0}$ (section 3.1); ii) when $\boldsymbol{E}_{s}=E_{s}^{z} \mathbf{k}=$ const, $\boldsymbol{B}_{s}=\mathbf{0}$ (section 3.2); iii) when $\boldsymbol{E}_{s}=\mathbf{0}$, $\boldsymbol{B}_{s}=B^{z} \mathbf{k}=$ const; iv) when $\boldsymbol{E}_{s}=E^{z} \mathbf{k}, \boldsymbol{B}_{s}=B^{z} \mathbf{k}$ are both nonzero constants (section 3.4); v) when $\boldsymbol{E}_{s}, \boldsymbol{B}_{s}$ are constant fulfilling the only condition $\boldsymbol{B}_{s}^{\perp}=\mathbf{k} \wedge \boldsymbol{E}_{s}^{\perp}$ (section 3.5).

\section{1 $E_{s}=B_{s}=0$, and the Lawson-Woodward theorem}

In the simplest case, $A_{s}^{\mu} \equiv 0$, not only $\hat{\Pi}^{\perp}$, but also $\hat{\Pi}^{z}$, and therefore $\hat{s}$, are constant, and (31-35) are solved by integration. The solution reads

$$
\begin{aligned}
& \hat{\boldsymbol{u}}^{\perp}(\xi)=\frac{q\left[\boldsymbol{K}^{\perp}-\boldsymbol{\alpha}^{\perp}(\xi)\right]}{m c^{2}}, \quad \hat{s}(\xi)=s_{0}, \\
& \hat{\gamma}(\xi)=\frac{1+\hat{\boldsymbol{u}}^{\perp 2}(\xi)+s_{0}^{2}}{2 s_{0}}, \quad \hat{u}^{z}(\xi)=\hat{\gamma}(\xi)-s_{0}, \\
& \hat{z}(\xi)=z_{0}+\frac{1-s_{0}^{2}}{2 s_{0}^{2}}\left(\xi-\xi_{0}\right)+\int_{\xi_{0}}^{\xi} d \zeta \frac{\hat{\boldsymbol{u}}^{\perp 2}(\zeta)}{2 s_{0}^{2}}, \\
& \hat{\boldsymbol{x}}^{\perp}(\xi)=\hat{\boldsymbol{x}}_{0}^{\perp}+\int_{\xi_{0}}^{\xi} d \zeta \frac{\hat{\boldsymbol{u}}^{\perp}(\zeta)}{s_{0}}, \quad \hat{t}(\xi)=\xi+\hat{z}(\xi) .
\end{aligned}
$$


These formulae can be obtained also solving the Hamilton-Jacobi equation $[4,25,26]^{6}$ in terms of the auxiliary parameter $\xi$, rather than on $t$; see also $[14,27]$. In appendix 5.3 we re-derive this result promoting $\xi$ to the new time variable, after having slightly generalized the machinery of canonical transformations to allow changes of the latter.

If the particle is at rest at the origin before the interaction with the wave, then $s_{0}=1$, $\boldsymbol{x}_{0}=\boldsymbol{K}^{\perp}=\mathbf{0}$, and (38) become

$$
\begin{array}{lcc}
\hat{s} \equiv 1, \quad \hat{\boldsymbol{u}}^{\perp}=\frac{-q \boldsymbol{\alpha}^{\perp}}{m c^{2}}, & \hat{u}^{z}=\frac{\hat{\boldsymbol{u}}^{\perp 2}}{2}, & \hat{\gamma}=1+\hat{u}^{z} \\
\hat{z}(\xi)=\int_{\xi_{0}}^{\xi} d \zeta \frac{\hat{\boldsymbol{u}}^{\perp 2}(\zeta)}{2}, & \hat{\boldsymbol{x}}^{\perp}(\xi)=\int_{\xi_{0}}^{\xi} d \zeta \hat{\boldsymbol{u}}^{\perp}(\zeta), & \hat{t}(\xi)=\xi+\hat{z}(\xi) .
\end{array}
$$

Remarks 3.1: As $u^{z} \geq 0$, the longitudinal motion is in all cases purely forward [the transverse one is oscillatory if $\boldsymbol{\epsilon}^{\perp}$ is of the type 2.2.1.1, see formula (21), or of the type 2.2.1.2]. Moreover, the maximum energy is attained at the maximum of $\alpha^{\perp}$; by (23), if the pump is slowly modulated this means approximately at the maximum of $\epsilon$. Note also that if we rescale $\boldsymbol{\epsilon}^{\perp} \mapsto a \boldsymbol{\epsilon}^{\perp}$ the transverse variables $\hat{\boldsymbol{x}}^{\perp}, \hat{\boldsymbol{u}}^{\perp}$ scale like $a$, whereas the longitudinal variables $\hat{z}, \hat{u}^{z}$ scale like $a^{2}$. The positive longitudinal drift and its quadratic scaling originate from the magnetic force $q \boldsymbol{\beta} \wedge \boldsymbol{B}$ (incidentally, the mean value of the latter over a cycle of carrier monochromatic wave is called the ponderomotive force): if e.g. $\boldsymbol{E}=\boldsymbol{\epsilon}^{\perp}=\epsilon^{x} \mathbf{i}$, then the motion is initially purelly oscillatory in the $x$ direction, but as the velocity grows then the magnetic force due to the magnetic field $\boldsymbol{B}=\epsilon^{x} \mathbf{j}$ deviates it also in the positive $z$ direction, so that the motion takes place in the $x z$ plane. Due to the mentioned scalings the trajectory goes to a straight line in the limit $a \rightarrow \infty$.

In fig. 2 we plot the solutions induced by a pulse modulated by a gaussian $\epsilon(\xi)=$ $a \exp \left[-\xi^{2} / 2 \sigma\right]$ for a couple of values of $a, \sigma$, and the corresponding trajectories.

Proposition 5 If $\boldsymbol{\epsilon}^{\perp}(\xi)$ goes to zero as $\xi \rightarrow \pm \infty$ the final 4-velocity and energy gain read

$$
\boldsymbol{u}_{f}^{\perp}=\hat{\boldsymbol{u}}^{\perp}(\infty), \quad u_{f}^{z}=\mathcal{E}_{f}=\frac{\boldsymbol{u}_{f}^{\perp 2}}{2}, \quad \gamma_{f}=1+\mathcal{E}_{f} ;
$$

if $\boldsymbol{\epsilon}^{\perp}(\xi)=0$ for $\xi \geq l$ these values are attained for all $\xi \geq l$.

If $\boldsymbol{\epsilon}^{\perp}$ is of the type (21) then $\boldsymbol{u}_{f}^{\perp}$ is a combination of the Fourier transform $\tilde{\epsilon}(k), \tilde{\epsilon}(-k)$. Therefore if $\epsilon$ is of the form (24) then $\boldsymbol{u}_{f}^{\perp} \rightarrow 0$ as $\eta \rightarrow \infty$ (by the Riemann-Lebesgue lemma, after integrating by parts); in particular, if $\epsilon_{0} \in \mathcal{S}(\mathbb{R})$, i.e. $\epsilon_{0}$ is smooth and fast decaying, then also $\tilde{\epsilon}(k), \boldsymbol{u}_{f}^{\perp} \in \mathcal{S}(\mathbb{R})$ and the decay as $\eta \rightarrow \infty$ is fast. From (40) it follows the

Corollary 2 If the electromagnetic field is a combination of terms of the form

$$
\boldsymbol{E}(\mathrm{x})=\boldsymbol{\epsilon}^{\perp}(c t-z), \quad \boldsymbol{B}(\mathrm{x})=\mathrm{k} \wedge \boldsymbol{\epsilon}^{\perp}(c t-z), \quad \boldsymbol{\epsilon}^{\perp}(\xi)=\epsilon(\xi) \boldsymbol{\epsilon}_{o}^{\perp}(\xi),
$$

with polarization vectors $\boldsymbol{\epsilon}_{o}^{\perp}$ of the form (21) and modulating amplitudes $\epsilon(\xi)$ going to zero as $\xi \rightarrow \pm \infty$ [in either form (19)], then the final energy gain $\mathcal{E}_{f}$ and variation $(\Delta \boldsymbol{u})_{f}$ of $\boldsymbol{u}$ go to zero if we rescale all $\epsilon$ as in (24) and let $\eta \rightarrow \infty$.

\footnotetext{
${ }^{6}$ Our $q, z, \xi, \boldsymbol{x}^{\perp}, s_{0}, \boldsymbol{K}^{\perp}, \boldsymbol{\alpha}^{\perp}$ are respectively denoted as $e, x, c \xi, \boldsymbol{r}, \gamma / m c, c \boldsymbol{f} / q, \boldsymbol{A}$ at pp. 128-129 of [4].
} 

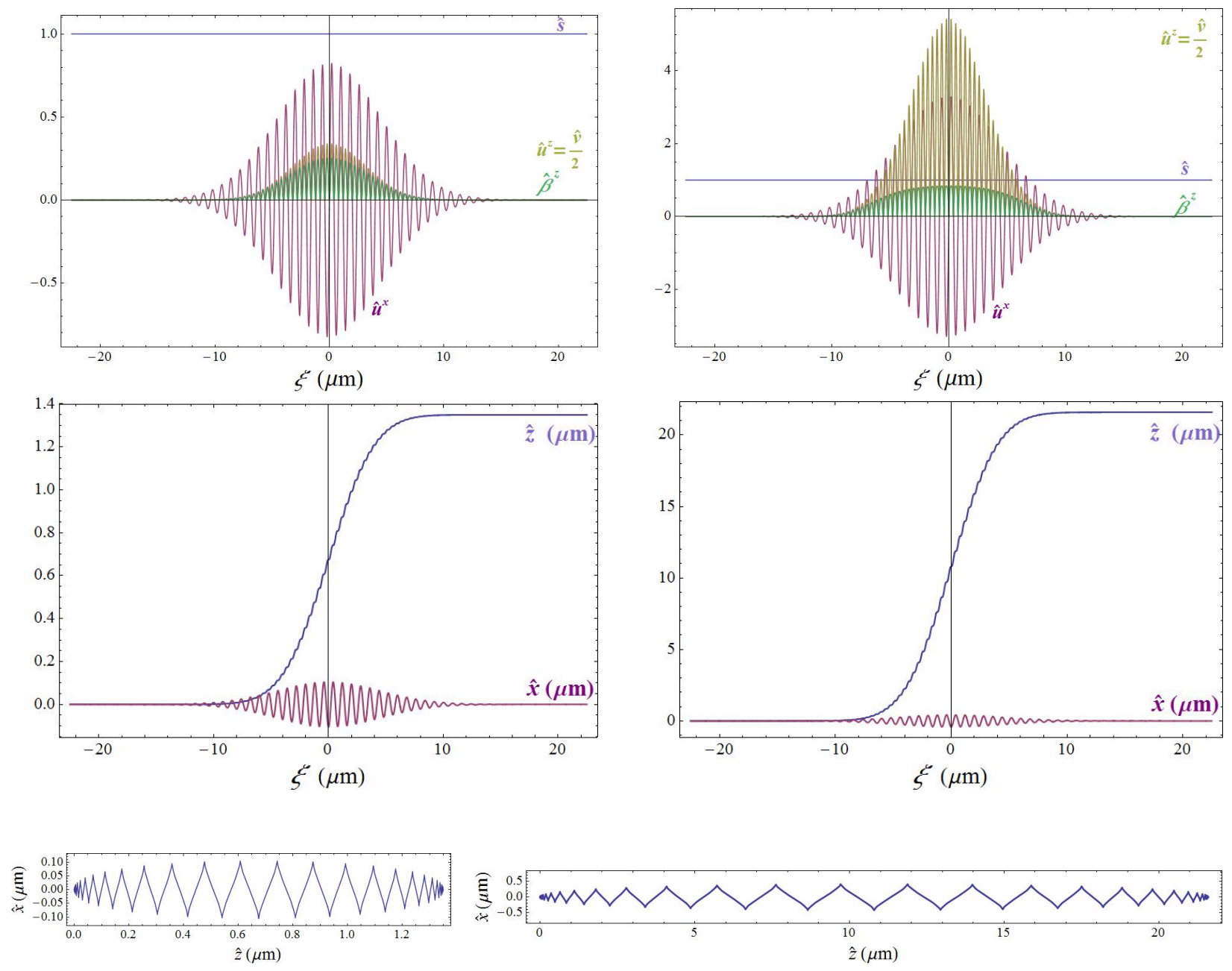

Figure 2: Left: the solution (39), (32) of (35), (8) (up, center) and the corresponding trajectory in the $z x$ plane (down) induced by a linearly polarized modulated pump (21-22) with wavelength $\lambda=2 \pi / k=0.8 \mu \mathrm{m}$, gaussian enveloping amplitude $\epsilon(\xi)=a \exp \left[-\xi^{2} / 2 \sigma\right]$ with $\sigma=20.3 \mu \mathrm{m}^{2}$ and $|q| a \sqrt{2} / k m c^{2}=0.8$, zero static fields $\left(\boldsymbol{E}_{s}=\boldsymbol{B}_{s}=\mathbf{0}\right)$ and trivial initial conditions $\left(\boldsymbol{x}_{0}=\boldsymbol{\beta}_{0}=0\right)$. Right: the solution (39), (32) (up, center) and the corresponding trajectory in the $z x$ plane (down) induced by a pump differing from the previous one only in the following parameter: $|q| a \sqrt{2} / k m c^{2}=3.3$. If the charged particle is an electron such parameters, or even sharper ones, can be easily achieved with present-day lasers. Correspondingly, the electron experiences huge accelerations: over distances of the order of half a micron, or - equivalently - over times of the order of 1 femtosecond, the $x$-component of the velocity changes from almost the velocity of light $c$ to almost the opposite $-c$, and viceversa; whereas the $z$ component changes form almost $c$ to zero, and viceversa. 
Proof. If the initial conditions are trivial the claim follows from (40), (23) and the results of appendix 5.4; if they are nontrivial it follows from the validity of the claim with respect to the inertial frame where the initial conditions are trivial.

We add that with respect to the latter frame (for sufficiently fast decay of $u^{\perp}$ ) the longitudinal displacement admits a finite limit $(\Delta z)_{f}=\lim _{\xi \rightarrow \infty} \int_{-\xi}^{\xi} d y \hat{\boldsymbol{u}}^{\perp 2}(y) / 2$. Note also that all $\epsilon$ can be made slowly varying (i.e. $\left|\epsilon^{\prime}\right| \ll|k \epsilon|$ ) by a sufficiently large (but finite) $\eta$; the corresponding small $\mathcal{E}_{f},(\Delta \boldsymbol{u})_{f}$ can be estimated by the results of appendix 5.4.

The above corollary is essentially the generalized Lawson-Woodward theorem of $[28]^{7}$. This is partly more and partly less general than the so-called Lawson-Woodward (LW) or (General) Acceleration Theorem $[29,33,31,30,32]$ (an outgrowth of the original Woodward-Lawson Theorem [41, 42]). The LW theorem states that, in spite of the large energy variations during the interaction, the final energy gain of a charged particle interacting with an electromagnetic field in vacuum is zero if:

1. the electromagnetic field is in vacuum with no static (neither electric or magnetic) part;

2. the particle is highly relativistic $(v \simeq c)$ along its whole path;

3. no walls or boundaries are present;

4. nonlinear (in the amplitude) effects due to the magnetic force $q \boldsymbol{\beta} \wedge \boldsymbol{B}$ are negligible;

5. the power radiated by the charged particle is negligible.

Condition 2 ensures that the motion is along a straight line (chosen as the $\vec{z}$-axis) with constant velocity $c$, independently of $\boldsymbol{E}$; the theorem was proven extending the claim from a monochromatic plane wave $\boldsymbol{E}$ to general $\boldsymbol{E}$ by linearity (the work done by the total electric force was the sum of the works done by its Fourier components), which was justified by condition (4). The claim can be justified also invoking quantum arguments (impossibility of absorption of a single real photon by 4-momentum conservation [32]), without need of assuming condition 2 .

Our Corollary 2 says that if we relax conditions 2, 4, but the electromagnetic field is a plane travelling wave, namely a superposition of very slowly modulated monochromatic ones, then we reach the same conclusion (no final energy or momentum variation).

To obtain a non-zero energy gain we need to violate one of the other conditions of the theorem, as we will do next.

\section{2 $\boldsymbol{E}_{s}=\boldsymbol{E}_{s}^{\|}=$const, $\boldsymbol{B}_{s}=0$ : acceleration, deceleration on a 'slope'}

Proposition 6 If $\boldsymbol{E}_{s}=E_{s}^{z} \mathbf{k}=$ const, $\boldsymbol{B}_{s}=\mathbf{0}$ and $\boldsymbol{\epsilon}^{\perp}(\xi)=0$ for $\left.\xi \notin\right] 0, l[$, then (35) is solved by

$$
\hat{s}(\xi)=s_{0}-\kappa \xi, \quad \hat{z}(\xi)=z_{0}+\frac{1}{2} \int_{0}^{\xi} d \zeta\left\{\frac{1+\hat{v}(\zeta)}{\left[s_{0}-\kappa \zeta\right]^{2}}-1\right\},
$$

\footnotetext{
${ }^{7}$ In $[28] \phi, \kappa, \boldsymbol{A}^{\perp}$ play the role of our $\xi, \hat{s}, \boldsymbol{u}^{\perp}$. Their assumption $\lim _{\phi \rightarrow \infty} \boldsymbol{A}^{\perp}(\phi)=0$ (in our words, $\boldsymbol{u}_{f}^{\perp}=0$ ) is to be understood as a physical statement valid with very good approximation in concrete experimental conditions $[39,40]$, rather than as a strict mathematical theorem. As an additional result, in [28] also the lowest correction to the above solution is computed using the Dirac-Lorentz equation.
} 
where $\kappa:=q E_{s}^{z} / m c^{2}$ and for simplicity we have chosen $\xi_{0}=0$. The other unknowns are obtained from formulas (30), (32-34).

If $\kappa>0$, (42) is well-defined only for $\xi<\xi_{f}:=s_{0} / \kappa$, because $(\hat{z}(\xi), \hat{s}(\xi)) \rightarrow(\infty, 0)$ as $\xi \rightarrow \xi_{f}$; but also in this case $(z(t), s(t))$ is defined for all $t$ (see remark point 2. in Remarks 2.2.1). Since $\hat{s}^{\prime} \equiv-\kappa$, the energy gain (37) from the beginning of the wave-particle interaction becomes

$$
\mathcal{E}=\int_{0}^{\xi_{1}} d \xi \frac{\hat{v}^{\prime}(\xi)}{2\left(s_{0}-\kappa \xi\right)}=\int_{0}^{\xi_{1}} d \xi \frac{-\kappa \hat{v}(\xi)}{2\left(s_{0}-\kappa \xi\right)^{2}}+\frac{\hat{v}\left(\xi_{1}\right)}{2\left(s_{0}-\kappa \xi_{1}\right)}
$$

The last term is negligible if $\boldsymbol{\epsilon}^{\perp}$ is a slowly modulated wave $(21)$ and $\epsilon(\xi)=0$ for $\xi \geq \xi_{1}$; hence $\mathcal{E}$ is positive if $\kappa<0$, negative if $\kappa>0$. Choosing $\xi_{1}=\xi_{f}$ in (43) we obtain the final energy gain $\mathcal{E}_{f}$ as a function of $\kappa$. If $\kappa \leq 0$ it is interesting to ask about the $\kappa_{M}$, if any, maximizing $\mathcal{E}_{f}$ for a given pump $\boldsymbol{\epsilon}^{\perp}$. If the latter is of the type (21), and $\epsilon$ varies slowly, has a unique maximum and vanishes at $\xi_{f}$, then there is a unique $\kappa_{M} \equiv q E_{s M}^{z} / m c^{2}$, determined by the equation $d \mathcal{E}_{f} / d \kappa=0$ (cf. fig. 3 left down). One can approximately realize an acceleration device of this kind as in fig 3 right: the particle initially lies at rest with $z_{0} \lesssim 0$, just at the left of a metallic grating $G$ contained in the $z=0$ plane and set at zero electric potential; another metallic grating $P$ contained in a plane $z=z_{p}>0$ is set at electric potential $V=V_{p}$. Then $E_{s}^{z}(z) \simeq 0$ for $z<0, E_{s}^{z}(z) \simeq-V_{p} / z_{p}$ for $0<z<z_{p}$. A short laser pulse $\boldsymbol{\epsilon}^{\perp}$ hitting the particle boosts it into the latter region through the ponderomotive force; choosing $q V_{p}>0$ implies $\kappa=-q V_{p} / z_{p} m c^{2}<0$, and a backward longitudinal electric force. If we choose $z_{p}>(\Delta z)_{f}$ (or $V$ large enough to avoid contact with $P$ ), then $z(t)$ will reach a maximum smaller than $z_{p}$, thereafter the particle will be accelerated backwards and will exit the grating with energy $\mathcal{E}_{f}$ and negligible transverse momentum, by point 3 . in Remarks 2.2.1. In other words, we obtain the same result as after kicking a ball initially at rest on a horizontal plane towards a hill: after climbing part of the slope the ball comes back to the initial position with nonzero velocity and flees away in the opposite direction. A large $\mathcal{E}_{f}$ requires very short and energetic laser pulses and extremely large $\left|V_{p}\right|$. With the presently available ultra-short and ultra-intense laser pulses the required $E_{s}^{z}$ to maximize $\mathcal{E}_{f}$ is far beyond the material breakdown threshold (namely, sparks between the plates arise and rapidly reduce their electric potential difference), what prevents its realization as a static field. Therefore in this form such an acceleration mechanism is little convenient from the practical viewpoint. A way out is to make the pulse itself generate such large $\left|E_{s}^{z}\right|$ within a plasma at the right time, as sketchily explained in section 4.1.

Similarly, one can approximately realize a deceleration device of this kind as in fig 3 downright: the particle initially moves backwards $\left(u^{z}<0, s_{0}>1\right)$, towards a metallic grating $G$ contained in the $z=0$ plane and set at zero electric potential; another metallic grating $P$ contained in a plane $z=-z_{p}<0$ is set at electric potential $V=V_{p}$. Then $E_{s}^{z}(z) \simeq 0$ for $z>0$, whereas $E_{s}^{z}(z) \simeq V_{p} / z_{p}$ for $-z_{p}<z<0$. Choosing $q V_{p}>0$ implies $\kappa=q V_{p} / z_{p} m c^{2}>0$, and a forward longitudinal electric force will brake the particle in the region $-z_{p}<z<0$; if in addition a short laser pulse $\boldsymbol{\epsilon}^{\perp}$ hits the particle inside the latter region, then the deceleration will be increased, due to the negative energy gain. 

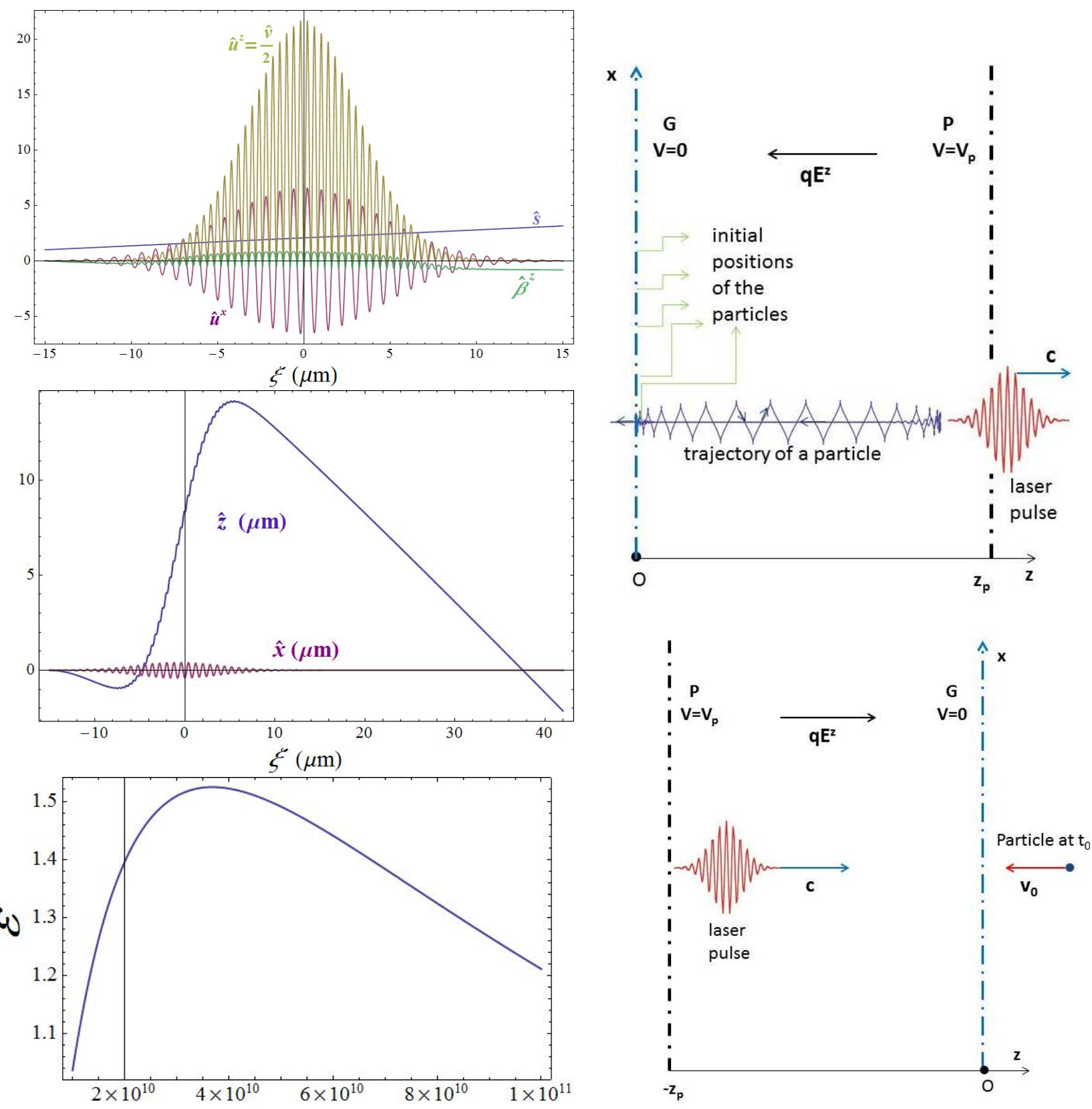

Figure 3: The solution (42), (32) (left up, left center) of (35), (8) induced by a linearly polarized modulated pump (21-22) with wavelength $\lambda=2 \pi / k=0.8 \mu \mathrm{m}$, gaussian enveloping amplitude $\epsilon(\xi)=a \exp \left[-\xi^{2} / 2 \sigma\right]$ with $\sigma=20.3 \mu \mathrm{m}^{2}$ and $|q| a \sqrt{2} / k m c^{2}=6.6$, trivial initial conditions, $\boldsymbol{B}_{s}=\mathbf{0}, \boldsymbol{E}_{s}=\mathbf{k} E_{M}^{z}$, where $E_{M}^{z} q \simeq 37 \mathrm{GeV} / \mathrm{m}$; right: the corresponding trajectory in the $z x$ plane within an hypothetical acceleration device based on a laser pulse and metallic gratings $G, P$ at potentials $V=0, V_{p}$, with $q V_{p} / z_{p} \simeq 37 \mathrm{GeV} / \mathrm{m}$. The chosen value $E_{M}^{z} \simeq$ $37 \mathrm{GV} / \mathrm{m}$ yields the maximum energy gain $\mathcal{E}_{f} \simeq 1.5$, as the graph of $\mathcal{E}_{f}$ vs. $E^{z}$ (left down) shows. Right down: hypothetical deceleration device based on a laser pulse and metallic gratings $G, P$ at potentials $V=0, V_{p}$, with $q V_{p}>0$. 


\section{3 $\boldsymbol{E}_{s}=0, \boldsymbol{B}_{s}=\boldsymbol{B}_{s}^{\|}=\mathrm{const}$, and cyclotron autoresonance}

Here we consider the case $\boldsymbol{E}_{s}=\boldsymbol{B}_{s}^{\perp}=\mathbf{0}$. By $(27) \hat{s}(\xi)=s_{0}$ and eq. (8) become

$$
\hat{x}^{\prime}=\frac{\hat{u}^{x}}{s_{0}}=w^{x}+b \hat{y}, \quad \hat{y}^{\prime}=\frac{\hat{u}^{y}}{s_{0}}=w^{y}-b \hat{x}, \quad \hat{z}^{\prime}=\frac{1+\hat{\boldsymbol{u}}^{\perp 2}}{2 s_{0}^{2}}-\frac{1}{2},
$$

where $b:=q B^{z} / s_{0} m c^{2}, \boldsymbol{w}^{\perp}(\xi):=\left[\boldsymbol{K}^{\perp}-\boldsymbol{\alpha}^{\perp}(\xi)\right] q / s_{0} m c^{2}$. If we combine the first two equations into the complex one

$$
(\hat{x}+i \hat{y})^{\prime}=-i b(\hat{x}+i \hat{y})+\left(w^{x}+i w^{y}\right),
$$

we immediately find the solution of the associated Cauchy problems; then $\hat{\boldsymbol{u}}, \hat{z}$ are found by derivation and integration using (44). Thus we arrive at

Proposition 7 If $\boldsymbol{E}_{s}=\mathbf{0}, \boldsymbol{B}_{s}=B_{s}^{z} \mathbf{k}=$ const the solution of the equations of motion reads

$$
\begin{aligned}
& (\hat{x}+i \hat{y})(\xi)=(\hat{x}+i \hat{y})\left(\xi_{0}\right)+\int_{\xi_{0}}^{\xi} d \zeta e^{-i b(\xi-\zeta)}\left(w^{x}+i w^{y}\right)(\zeta) . \\
& \hat{s}(\xi)=s_{0}, \quad \hat{\boldsymbol{u}}^{\perp}=s_{0} \hat{\boldsymbol{x}}^{\perp,}, \quad \hat{u}^{z}=s_{0} \hat{z}^{\prime}=\frac{1+\hat{\boldsymbol{u}}^{\perp 2}}{2 s_{0}}-\frac{s_{0}}{2}, \\
& \hat{z}(\xi)=\hat{z}\left(\xi_{0}\right)+\int_{\xi_{0}}^{\xi} \zeta\left[\frac{1+\hat{\boldsymbol{u}}^{\perp 2}(\zeta)}{2 s_{0}^{2}}-\frac{1}{2}\right] .
\end{aligned}
$$

Formulae (46) give the exact solution. Using (44) one easily finds that $\partial \hat{v} / \partial \xi=\hat{v}^{\prime}$,

$$
\hat{v}^{\prime}-\frac{\partial \hat{v}}{\partial \xi}=\hat{\boldsymbol{u}}^{\perp} \cdot\left(\hat{\boldsymbol{u}}^{\perp \prime}-\frac{\partial \hat{\boldsymbol{u}}^{\perp}}{\partial \xi}\right)=\hat{u}^{x}(b \hat{y})^{\prime}+\hat{u}^{y}(-b \hat{x})^{\prime}=\frac{b}{s_{0}}\left(\hat{u}^{x} \hat{u}^{y}-\hat{u}^{y} \hat{u}^{x}\right)=0,
$$

so that the exact energy gain is $\mathcal{E}(\xi)=\int_{\xi_{0}}^{\xi} d y \hat{v}^{\prime}(y) / 2 s_{0}=\left[\hat{v}(\xi)-\hat{v}\left(\xi_{0}\right)\right] / 2 s_{0}$. In particular, if the particle starts at rest from the origin at $t=0$, then $\boldsymbol{x}(0)=\mathbf{0}=\boldsymbol{u}(0)=\boldsymbol{K}^{\perp}, s_{0} \equiv 1$, and

$$
\begin{aligned}
& (\hat{x}+i \hat{y})(\xi)=\int_{0}^{\xi} d \zeta e^{-i b(\xi-\zeta)}\left(w^{x}+i w^{y}\right)(\zeta), \quad \hat{\boldsymbol{u}}^{\perp}=\hat{\boldsymbol{x}}^{\perp \prime}, \\
& \hat{u}^{z}=\hat{z}^{\prime}=\frac{\hat{\boldsymbol{u}}^{\perp 2}}{2}=\mathcal{E}(\xi)=\hat{\gamma}(\xi)-1, \quad \hat{z}(\xi)=\int_{0}^{\xi} d \zeta \frac{\hat{\boldsymbol{u}}^{\perp 2}(\zeta)}{2} .
\end{aligned}
$$

In appendix 5.5 we show that in the limit of a monochromatic pump our solution (47) reduces to the approximate one found in $[23,24,45]$ and (up to our knowledge) in the rest

of the literature. We also recall how to tune $\boldsymbol{B}_{s}=B^{z} \mathbf{k}$ so that the acceleration by the pulse becomes resonant, and the quantitative features of this mechanism (cyclotron autoresonance). We emphasize that instead our solution (47) is exact for all pumps $\boldsymbol{\epsilon}^{\perp}$, and with it one can also determine the deviations from autoresonance due to an arbitrary modulation (21-22) of the monochromatic pulse.

\subsection{Constant longitudinal $\boldsymbol{E}_{s}=\boldsymbol{E}_{s}^{\|}, \boldsymbol{B}_{s}=\boldsymbol{B}_{s}^{\|}$}

If also $E_{s}^{z} \neq 0$, then by (27) $\hat{s}(\xi)=s_{0}-\kappa \xi$ and eq. (8) become

$$
\hat{x}^{\prime}=\frac{\hat{u}^{x}}{\hat{s}}=\frac{w^{x}+b \hat{y}}{\hat{s}}, \quad \hat{y}^{\prime}=\frac{\hat{u}^{y}}{\hat{s}}=\frac{w^{y}-b \hat{x}}{\hat{s}}, \quad \hat{z}^{\prime}=\frac{1+\hat{\boldsymbol{u}}^{\perp 2}}{2 \hat{s}^{2}}-\frac{1}{2},
$$


where again $\kappa:=q E_{s}^{z} / m c^{2}, b:=q B^{z} / m c^{2}, \boldsymbol{w}^{\perp}(\xi):=\left[\boldsymbol{K}^{\perp}-\boldsymbol{\alpha}^{\perp}(\xi)\right] q / m c^{2}$. Arguing as before we can prove

Proposition 8 If $\boldsymbol{E}_{s}=E_{s}^{z} \mathbf{k}, \boldsymbol{B}_{s}=B_{s}^{z} \mathbf{k}$ are constant the solution of the equations of motion reads

$$
\begin{aligned}
& (\hat{x}+i \hat{y})(\xi)=\left(s_{0}-\kappa \xi\right)^{i b / \kappa}\left[\frac{(\hat{x}+i \hat{y})\left(\xi_{0}\right)}{\left(s_{0}-\kappa \xi_{0}\right) i b / \kappa}+\int_{\xi_{0}}^{\xi} d \zeta \frac{\left(w^{x}+i w^{y}\right)(\zeta)}{\left(s_{0}-\kappa \zeta\right)^{1+i b / \kappa}}\right], \\
& \hat{s}(\xi)=s_{0}-\kappa \xi, \quad \hat{u}^{z}(\xi)=\frac{1}{2\left(s_{0}-\kappa \xi\right)}+\left(s_{0}-\kappa \xi\right) \frac{\hat{\boldsymbol{x}}^{\perp / 2}(\xi)-1}{2}, \\
& \hat{\boldsymbol{u}}^{\perp}(\xi)=\left(s_{0}-\kappa \xi\right) \hat{\boldsymbol{x}}^{\perp \prime}(\xi), \quad \hat{\gamma}(\xi)=s_{0}-\kappa \xi+\hat{u}^{z}(\xi), \\
& \hat{z}(\xi)=\hat{z}\left(\xi_{0}\right)+\int_{\xi_{0}}^{\xi} \frac{d \zeta}{2}\left[\frac{1}{\left(s_{0}-\kappa \zeta\right)^{2}}+\hat{\boldsymbol{x}}^{\perp \prime 2}(\zeta)-1\right] .
\end{aligned}
$$

Note that this reduces to (46) in the limit $\kappa \rightarrow 0$, and again $\partial \hat{v} / \partial \xi=\hat{v}^{\prime}$. In the case of initial conditions $\boldsymbol{x}(0)=\mathbf{0}=\boldsymbol{u}(0)$ then (49) becomes

$$
\begin{aligned}
& (\hat{x}+i \hat{y})(\xi)=(1-\kappa \xi)^{i b / \kappa} \int_{0}^{\xi} d \zeta \frac{\left(w^{x}+i w^{y}\right)(\zeta)}{(1-\kappa \zeta)^{1+i b / \kappa}}, \quad \hat{s}(\xi)=1-\kappa \xi \\
& \hat{z}(\xi)=\int_{0}^{\xi} \frac{d \zeta}{2}\left[\frac{1}{(1-\kappa \zeta)^{2}}+\hat{\boldsymbol{x}}^{\perp \prime 2}(\zeta)-1\right], \quad \hat{\boldsymbol{u}}^{\perp}(\xi)=(1-\kappa \xi) \hat{\boldsymbol{x}}^{\perp \prime}(\xi), \\
& \hat{u}^{z}(\xi)=\frac{1}{2(1-\kappa \xi)}+(1-\kappa \xi) \frac{\hat{\boldsymbol{x}}^{\perp \prime 2}(\xi)-1}{2}, \quad \hat{\gamma}(\xi)=1-\kappa \xi+\hat{u}^{z}(\xi) .
\end{aligned}
$$

\subsection{Adding constant $\boldsymbol{E}_{s}^{\perp}$ and $\boldsymbol{B}_{s}^{\perp}=\mathrm{k} \wedge \boldsymbol{E}_{s}^{\perp}$ to $\boldsymbol{E}_{s}^{\|}, \boldsymbol{B}_{s}^{\|}$}

Proposition 9 If $\boldsymbol{E}_{s}, \boldsymbol{B}_{s}$ are constant fulfilling the only condition $\boldsymbol{B}_{s}^{\perp}=\mathbf{k} \wedge \boldsymbol{E}_{s}^{\perp}$ then the solutions take the form (49-50), with $\boldsymbol{w}^{\perp}(\xi):=q\left[\boldsymbol{K}^{\perp}-\boldsymbol{\alpha}^{\perp}(\xi)+\xi \boldsymbol{E}_{s}^{\perp}\right] / m c^{2}, \quad b:=q B_{s}^{z} / m c^{2}$, $\kappa:=q E_{s}^{z} / m c^{2}$. In particular, if $E_{s}^{z}=0$ then $\hat{s}=s_{0}=$ const and they reduce to (46-47).

Proof: Choosing the reference frame so that $\boldsymbol{E}_{s}^{\perp}=\mathbf{i} E_{s}^{\perp}, \boldsymbol{B}_{s}^{\perp}=\mathbf{j} E_{s}^{\perp}$, (28) yields

$$
\hat{\boldsymbol{u}}^{\perp}=\boldsymbol{w}^{\perp}(\xi)+b(\mathbf{i} \hat{y}-\mathbf{j} \hat{x}), \quad \hat{s}(\xi)=\frac{-q}{m c^{2}}\left[K^{z}+\xi E_{s}^{z}\right] \equiv s_{0}-\kappa \xi
$$

These formulas show that eq. (8) take again the linear form (48). Then eq. (49-50) apply. In particular if $E_{s}^{z}=0$ then $\hat{s}=$ const and eq. (46-47) apply.

Up to our knowledge the solutions with $\boldsymbol{E} \neq \mathbf{0}$ have not appeared in the literature before.

\section{Plasmas in the hydrodynamic approximation}

For a system of many charged particles in an external EM field the Action and the Lagrangian take the form

$$
\hat{\boldsymbol{S}}_{m}=\int_{\xi_{0}}^{\xi_{1}} \frac{d \xi}{c} \sum_{\alpha} \mathcal{L}\left[\hat{\boldsymbol{x}}_{\alpha}, \hat{\boldsymbol{x}}_{\alpha}^{\prime}, \xi ; m_{\alpha}, q_{\alpha}\right]
$$


where index $\alpha$ enumerates the particle, and $m_{\alpha}, q_{\alpha}$ are the mass and charge of the $\alpha$-th particle. If the number of particles of the same species in every macroscopic volume element $d V$ in the physical $\boldsymbol{x}$-space is huge, and these particles approximately have the same velocity - as within a plasma in hydrodynamic conditions - we can macroscopically describe these particles by a fluid. In the Lagrangian description the previous formula then becomes

$$
\begin{aligned}
\boldsymbol{S}_{m} & =\int_{\xi_{0}}^{\xi_{1}} \frac{d \xi}{c} \int d \boldsymbol{X} \hat{\boldsymbol{L}}_{m}\left[\left\{\hat{\boldsymbol{x}}_{h}(\xi, \boldsymbol{X})\right\},\left\{\hat{\boldsymbol{x}}_{h}^{\prime}(\xi, \boldsymbol{X})\right\} ; \xi\right], \\
\hat{\boldsymbol{L}}_{m} & :=\sum_{h} \widetilde{n_{h 0}}(\boldsymbol{X})\left[-m_{h} c^{2} \sqrt{1+2 \hat{z}_{h}^{\prime}-\hat{\boldsymbol{x}}_{h}^{\perp \prime 2}}-q_{h}\left(1+\hat{z}_{h}^{\prime}\right) A^{0}\left(\xi+\hat{z}_{h}, \hat{\boldsymbol{x}}_{h}\right)+q_{h} \hat{\boldsymbol{x}}_{h}^{\prime} \cdot \boldsymbol{A}\left(\xi+\hat{z}_{h}, \hat{\boldsymbol{x}}_{h}\right)\right] .
\end{aligned}
$$

Here $h$ enumerates the particle species, $m_{h}, q_{h}$ are the $h$-th rest mass and charge, the prime denotes now partial differentiation with respect to $\xi, \boldsymbol{X}$ is an auxiliary vector variable (like the initial position) used to distinguish the material fluid elements, $\widetilde{n_{h 0}}(\boldsymbol{X})$ is the associated density (number of particles per unit volume $d \boldsymbol{X}$ ) of the $h$-th fluid; together with the EM field, the $\widetilde{n_{h 0}}(\boldsymbol{X})$ are part of the assigned data. $\boldsymbol{x}_{h}(t, \boldsymbol{X})$ is the position at time $t$ of the material element (of the $h$-th fluid) identified by $\boldsymbol{X}, \hat{\boldsymbol{x}}_{h}(\xi, \boldsymbol{X})$ the position of the same material element as a function of $\xi$. The function $\boldsymbol{x}_{h}$ is required to have continuous second derivatives (at least piecewise) and for every $t$ the restriction $\boldsymbol{x}_{h}(t, \cdot): \boldsymbol{X} \mapsto \boldsymbol{x}$ is required to be one-to-one. Equivalently, $\hat{\boldsymbol{x}}_{h}$ is required to have continuous second derivatives (at least piecewise) and for every $\xi$ the map $\hat{\boldsymbol{x}}_{h}(\xi, \cdot): \boldsymbol{X} \mapsto \boldsymbol{x}$ is required to be one-to-one; the equivalence holds because both conditions of "being one-to-one" amount to the condition that "no two different particle-worldlines intersect" (see fig. 1 right). For every $t$ we denote as $\boldsymbol{X}_{h}(t, \cdot): \boldsymbol{x} \mapsto \boldsymbol{X}$ the inverse of $\boldsymbol{x}_{h}(t, \cdot)$, and for every $\xi$ we denote as $\hat{\boldsymbol{X}}_{h}(\xi, \cdot): \boldsymbol{x} \mapsto \boldsymbol{X}$ the inverse of $\hat{\boldsymbol{x}}_{h}(\xi, \cdot)$. Clearly,

$$
\boldsymbol{X}_{h}(t, \boldsymbol{x})=\hat{\boldsymbol{X}}_{h}(c t-z, \boldsymbol{x}), \quad \operatorname{det}\left(\frac{\partial \boldsymbol{X}_{h}}{\partial \boldsymbol{x}}\right)=\operatorname{det}\left(\frac{\partial \hat{\boldsymbol{X}}_{h}(c t-z, \boldsymbol{x})}{\partial \boldsymbol{x}}\right) .
$$

The Jacobians $J_{h}:=\operatorname{det}\left(\frac{\partial \boldsymbol{x}_{h}}{\partial \boldsymbol{X}}\right), \hat{J}_{h}:=\operatorname{det}\left(\frac{\partial \hat{\boldsymbol{x}}_{h}}{\partial \boldsymbol{X}}\right)$ are the inverses of the left and right determinants (expressed in terms of the appropriate independent variables), respectively. We denote as $n_{h}(t, \boldsymbol{x})$ the Eulerian density of the $h$-fluid. In the ( $\xi$-parametrized) Lagrangian and in the ( $t$-parametrized) Eulerian description the conservation of the number of particles of the $h$-th fluid in every material volume element $d \boldsymbol{X}$ respectively amount to

$$
\hat{n}_{h}(\xi, \boldsymbol{X}) \hat{J}_{h}(\xi, \boldsymbol{X})=\widetilde{n_{h 0}}(\boldsymbol{X}) \quad \Leftrightarrow \quad n_{h}(t, \boldsymbol{x})=\left\{\widetilde{n_{h 0}}\left[\hat{\boldsymbol{X}}_{h}(\xi, \boldsymbol{x})\right] \hat{J}_{h}^{-1}(\xi, \boldsymbol{x})\right\}_{\xi=c t-z},
$$

which allow to compute $\hat{n}_{h}(\xi, \boldsymbol{X}), n_{h}(t, \boldsymbol{x})$ after having solved the other equations.

The Hamiltonian expressed as a function of the $\hat{\boldsymbol{x}}_{h}, \hat{\boldsymbol{\Pi}}_{h}:=\partial \boldsymbol{L}_{m} / \partial \hat{\boldsymbol{x}}_{h}^{\prime}$ reads

$$
\hat{\boldsymbol{H}}\left(\left\{\hat{\boldsymbol{x}}_{h}\right\},\left\{\hat{\boldsymbol{\Pi}}_{h}\right\} ; \xi\right)=\int d \boldsymbol{X} \sum_{h} \widetilde{n_{h 0}}(\boldsymbol{X}) \hat{H}\left(\hat{\boldsymbol{x}}_{h}, \hat{\boldsymbol{\Pi}}_{h}, \xi ; m_{h}, q_{h}\right)
$$

with $\hat{H}$ as defined in (12). The unknowns $\hat{\boldsymbol{x}}_{h}(\xi, \boldsymbol{X}), \hat{\boldsymbol{u}}_{h}(\xi, \boldsymbol{X})$ fulfill the associated Hamilton equations, which are a family (parametrized by the index $h$ and the argument $\boldsymbol{X}$ ) of systems of equations of the form (8), (14). 
To generalize our framework to a generic plasma according to kinetic theory one should consider $\boldsymbol{X}$ as a vector in 6 -dim phase space $[\boldsymbol{X}$ could be the pair of the initial $(\hat{\boldsymbol{x}}, \hat{\boldsymbol{\Pi}})]$, introduce corresponding densities in phase space and $\int d \boldsymbol{X}$ as integration over the latter.

If the back-reaction of the charged fluids on the EM is not negligible, then $A^{\mu}$ (or better its non-gauge, physical degrees of freedom) become unknown themselves, ruled by the Maxwell equations

$$
\square A^{\nu}-\partial^{\nu}\left(\partial_{\mu} A^{\mu}\right)=\partial_{\mu} F^{\mu \nu}=4 \pi j^{\nu},
$$

which can be obtained as Euler-Lagrange equations by variation with respect to $A^{\mu}$ of the action

$$
\boldsymbol{S}=\boldsymbol{S}_{m}+\boldsymbol{S}_{A}, \quad \boldsymbol{S}_{A}=\int d \Omega \frac{1}{16 \pi} F^{\mu \nu} F_{\mu \nu}
$$

( $\boldsymbol{S}_{A}$ is the action of the EM field, $d \Omega$ is the volume element in Minkowski space ), or the equivalent associated Hamilton equations for the unknowns $\boldsymbol{B}, \boldsymbol{E}$. Eq. (57) couple the EM field to the fluid motion through the current density $\left(j^{\mu}\right)=\sum_{h}\left(j_{h}^{\mu}\right)=\sum_{h}\left(j_{h}^{0}, \mathbf{j}_{h}\right)$, given by $j_{h}^{0}=q_{h} n_{h}, \quad \mathbf{j}_{h}=q_{h} n_{h} \boldsymbol{v}_{h} / c=q_{h} n_{h} \boldsymbol{\beta}_{h}$, with the $n_{h}$ as defined in (55) and

$$
\boldsymbol{\beta}_{h}(t, \boldsymbol{x})=\hat{\boldsymbol{\beta}}_{h}\left[c t-z, \hat{\boldsymbol{X}}_{h}(c t-z, \boldsymbol{x})\right] .
$$

Each current density $j_{h}^{\mu}$, and therefore also the total one $j^{\mu}$, are conserved: $\partial_{\mu} j_{h}^{\mu}=0$, etc. In the Landau gauge (57) simplifies to $\square A^{\nu}=4 \pi j^{\nu}$. In the Eulerian description the action functional (58) takes the form

$$
\boldsymbol{S}=\int d \Omega\left[-\sum_{h} \frac{m_{h} c^{2} n_{h}}{\gamma_{h}}+j^{\mu} A_{\mu}+\frac{1}{16 \pi} F^{\mu \nu} F_{\mu \nu}\right] \equiv \int d \Omega \boldsymbol{L}(\mathrm{x})
$$

\subsection{Plane problems. EM wave hitting a plasma at equilibrium}

The above formalism is useful in plane problems, i.e. if all the initial (or $t \rightarrow-\infty$ asymptotic) data [velocities, densities, EM fields of the form (17)] do not depend on the transverse coordinates. Then also the solutions for $\boldsymbol{B}, \boldsymbol{E}, \boldsymbol{u}_{h}, n_{h}$, the displacements $\Delta \boldsymbol{x}_{h}(t, \boldsymbol{X})$ and their hatted counterparts will not depend on them.

Here we consider more specifically the problem of the impact of an EM plane wave on a plasma initially in equilibrium. We therefore assume that for $t \leq 0$ : all fluids are at rest with densities vanishing in the region $z<0$ and summing up to a vanishing total electric density everywhere; that the EM field is of the form (17) with zero static electric field (for simplicity), constant static magnetic field $\boldsymbol{B}_{s}$, and pump (19a) with support contained in some interval $[0, l]$, so that at $t=0$ the wave (travelling in the positive $z$ direction) has not reached the plasma yet. This amounts to assume as $t=0$ initial conditions

$$
\begin{array}{lrrr}
\boldsymbol{u}_{h}(0, \boldsymbol{x})=\mathbf{0}, & n_{h}(0, \boldsymbol{x})=0 & \text { if } z \leq 0, & j^{0}(0, \boldsymbol{x})=\sum_{h} q_{h} n_{h}(0, \boldsymbol{x}) \equiv 0, \\
\boldsymbol{E}(0, \boldsymbol{x})=\boldsymbol{\epsilon}^{\perp}(-z), & \boldsymbol{B}(0, \boldsymbol{x})=\mathbf{k} \wedge \boldsymbol{\epsilon}^{\perp}(-z)+\boldsymbol{B}_{s}, & \boldsymbol{\epsilon}^{\perp}(\xi)=0 & \text { if } \xi \notin] 0, l[.
\end{array}
$$


These are compatible with the following initial conditions for the gauge potential:

$$
\partial_{t} \boldsymbol{A}(0, \boldsymbol{x})=-c \boldsymbol{\epsilon}^{\perp}(-z), \quad \boldsymbol{A}(0, \boldsymbol{x})=\boldsymbol{\alpha}^{\perp}(-z)+\boldsymbol{B}_{s} \wedge \boldsymbol{x} / 2,
$$

with $\boldsymbol{\alpha}^{\perp}$ as defined in $(20) ; \boldsymbol{\alpha}^{\perp}(\xi)=\mathbf{0}$ if $\xi \leq 0$. We choose $\boldsymbol{X} \equiv(X, Y, Z)$ as the $(t=0)$ initial position of the generic material element of the $h$-th fluid; $\boldsymbol{x}_{h}(t, \boldsymbol{X})$ will be its position at time $t$, etc. Consequently, $\widetilde{n_{h 0}}(Z)=n_{h}(0, Z)$. We denote as $\boldsymbol{x}_{h} \equiv\left(x_{h}, y_{h}, z_{h}\right), \hat{\boldsymbol{x}}_{h} \equiv\left(\hat{x}_{h}, \hat{y}_{h}, \hat{z}_{h}\right)$, $\boldsymbol{X}_{h} \equiv\left(X_{h}, Y_{h}, Z_{h}\right), \hat{\boldsymbol{X}}_{h} \equiv\left(\hat{X}_{h}, \hat{Y}_{h}, \hat{Y}_{h}\right)$ the components of these functions and of their inverses in the $\mathbf{i}, \mathbf{j}, \mathbf{k}$ basis.

Due to the dependence only on the longitudinal coordinate, (54) yields $Z_{h}(t, z)=\hat{Z}_{h}(c t-$ $z, z), \partial_{z}\left[\hat{Z}_{h}(c t-z, z)\right]=\partial_{z} Z_{h}(t, z)$, and (55b) simplifies to

$$
n_{h}(t, z)=n_{h 0}(t, z) \partial_{z} Z_{h}(t, z), \quad \text { where } n_{h 0}(t, z):=\widetilde{n_{h 0}}\left[\hat{Z}_{h}(c t-z, z)\right] .
$$

$\partial_{t} Z=0$ in the Eulerian description becomes $\frac{d Z_{h}}{d t}=\partial_{t} Z_{h}+v_{h}^{z} \partial_{z} Z_{h}=0$, which by (63) gives

$$
n_{h 0} \partial_{t} Z_{h}+n_{h} v_{h}^{z}=0 .
$$

Another important simplification is that we can solve [14] the Maxwell equations

$$
\nabla \cdot \boldsymbol{E}=\partial_{z} E^{z}=4 \pi j^{0}, \quad \partial_{0} E^{z}+4 \pi j^{z}=(\nabla \wedge \boldsymbol{B})^{z}=0
$$

for $E^{z}$ explicitly in terms of the assigned initial densities and of the unknowns $Z_{h}(t, z)$; thereby the number of unknowns is reduced. In fact, let $\widetilde{N}_{h}(Z):=\int_{0}^{Z} d Z^{\prime} \widetilde{n_{h 0}}\left(Z^{\prime}\right)$ be the number of particles of the $h$-th species per unit surface in the layer $0 \leq Z^{\prime} \leq Z$. Note that from (61) it follows $\sum_{h} q_{h} \widetilde{N}_{h}(Z) \equiv 0$. Setting $N_{h}(t, z):=\widetilde{N}_{h}\left[Z_{h}(t, z)\right]$, by (63-64) one immediately finds ${ }^{8}$

$$
\partial_{z} N_{h}=n_{h}, \quad \partial_{t} N_{h}=-n_{h} v_{h}^{z} .
$$

This implies that (Proposition 1 in [14]) eq. (65), (61) are solved by

$$
E^{z}(t, z)=4 \pi \sum_{h} q_{h} \widetilde{N}_{h}\left[Z_{h}(t, z)\right], \quad \widetilde{N}_{h}(Z):=\int_{0}^{Z} d Z^{\prime} \widetilde{n_{h 0}}\left(Z^{\prime}\right) .
$$

By (61-62) and causality it follows that $\boldsymbol{x}_{h}(t, \boldsymbol{X})=\boldsymbol{X}, \boldsymbol{A}^{\perp}(t, \boldsymbol{x}) \equiv \boldsymbol{B}_{s} \wedge \boldsymbol{x} / 2$ if $c t \leq z$, and $\mathbf{j} \equiv \mathbf{0}$ if $c t \leq|z|$; the transverse component of eq. (57) and (62) are equivalent to the integral equation (for $t \geq 0$ )

$$
\begin{aligned}
\boldsymbol{A}^{\perp}(t, \boldsymbol{x})-\boldsymbol{\alpha}^{\perp}(c t-z)-\frac{\boldsymbol{B}_{s}}{2} \wedge \boldsymbol{x} & =4 \pi c \int d t^{\prime} d z^{\prime} G\left(t-t^{\prime}, z-z^{\prime}\right) \theta\left(t^{\prime}\right) \boldsymbol{j}^{\perp}\left(t^{\prime}, z^{\prime}\right) \\
& =2 \pi c \int_{T \cap D_{t, z}} d t^{\prime} z^{\prime} \boldsymbol{j}^{\perp}\left(t^{\prime}, z^{\prime}\right)
\end{aligned}
$$

here $2 G(t, z)=\theta(c t-|z|)$ (the characteristic function of the 2-dim causal cone $T=\{(t, z) \mid c t>$ $|z|\})$ is used to express the Green function of the d'Alembertian $\partial_{0}^{2}-\partial_{z}^{2}$, and $D_{t, z}=\left\{\left(t^{\prime}, z^{\prime}\right) \mid c t-\right.$

\footnotetext{
${ }^{8} \mathrm{In}$ fact, $\partial_{z} N_{h}(t, z)=\left(\partial_{z} Z_{h}\right) \partial_{Z} \widetilde{N}_{h}\left[Z_{h}(t, z)\right]=\left(\partial_{z} Z_{h}\right) \widetilde{n_{h 0}}\left[Z_{h}(t, z)\right]=n_{h}(t, z), \quad \partial_{t} N_{h}(t, z)=$ $\left(\partial_{t} Z_{h}\right) \partial_{Z} \widetilde{N}_{h}\left[Z_{h}(t, z)\right]=\left(\partial_{t} Z_{h}\right) \widetilde{n_{h 0}}\left[Z_{h}\left(x^{0}, z\right)\right]=-\left(n_{h} v_{h}\right)(t, z)$.
} 


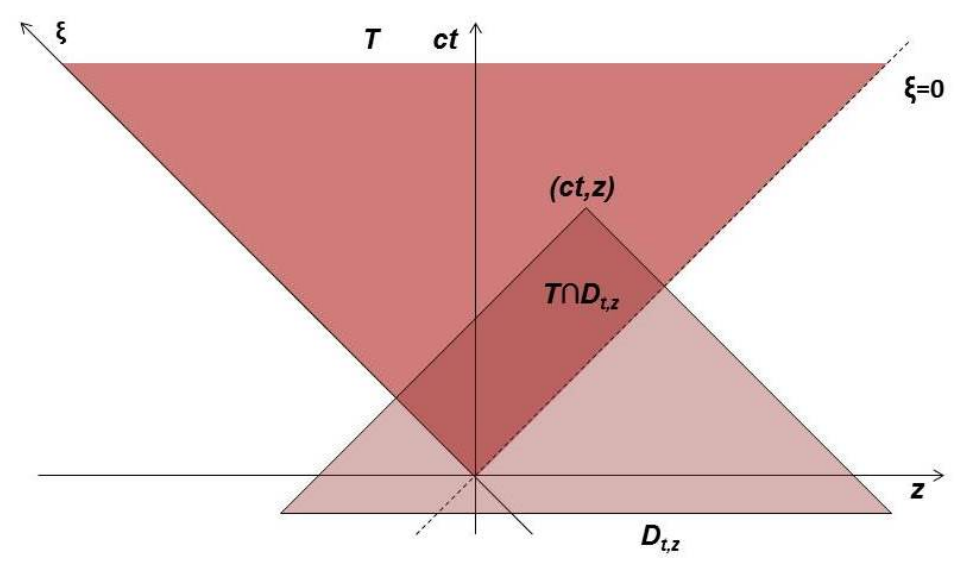

Figure 4: The 2-dim future causal cone $T=\{(t, z)|c t>| z \mid\}$ of the origin, the past causal cone $D_{t, z}=\left\{\left(t^{\prime}, z^{\prime}\right)\left|c t-c t^{\prime}>\right| z-z^{\prime} \mid\right\}$ of the point $(t, z) \in T$, and their intersection.

$\left.c t^{\prime}>\left|z-z^{\prime}\right|\right\} . D_{t, z} \cap T$ is empty if $t \leq 0$ or $c t \leq z$, a rectangle as in fig. 4 otherwise. If $\boldsymbol{\alpha}^{\perp}$ is large (or the densities are small) we can neglect the right-hand side of (68) and thus consider $\boldsymbol{A}^{\perp}=\boldsymbol{\alpha}^{\perp}+\boldsymbol{B}_{s} \wedge \boldsymbol{x} / 2$ and $\boldsymbol{E}, \boldsymbol{B}$ of the form (17) also for small positive times; the spacetime region in which such an approximation is acceptable can be determined a posteriori. Then the equations of motion for the fluids take the form of the families - parametrized by the argument $Z$ and the index $h-(8),(25)$, where $\boldsymbol{E}_{s}^{\perp}=\mathbf{0}, \boldsymbol{B}_{s}=$ const and $E_{s}^{z}$ is replaced by (67); the latter introduces a coupling among the motions of the different fluids.

For small times we can also neglect the motion of ions with respect to that of the much lighter electrons, and therefore consider their densities as static. By the initial electric neutrality of the plasma the initial proton density (due to ions of all kinds) equals the initial electron density, which we denote simply as $\widetilde{n_{0}}(Z)$. The longitudinal electric field thus depends on $t$ (resp. on $\xi$ ) only through the longitudinal coordinates of the electrons, and (67) becomes

$$
E^{z}(t, z)=4 \pi e\left\{\tilde{N}(z)-\tilde{N}\left[Z_{e}(t, z)\right]\right\} \quad \Leftrightarrow \quad \hat{E}^{z}(\xi, Z)=4 \pi e\left\{\tilde{N}\left[\hat{z}_{e}(\xi, Z)\right]-\tilde{N}(Z)\right\}
$$

with $\tilde{N}(Z):=\int_{0}^{Z} d Z^{\prime} \widetilde{n_{0}}\left(Z^{\prime}\right)$, and the longitudinal electric force acting on the $Z$ electrons is $\widetilde{F_{e}^{z}}(t, Z) \equiv F_{e}^{z}\left[z_{e}(t, Z), Z\right]\left(\operatorname{resp} . \hat{F}_{e}^{z}(\xi, Z) \equiv F_{e}^{z}\left[\hat{z}_{e}(\xi, Z), Z\right]\right)$, where

$$
F_{e}^{z}(z, Z):=-e E^{z}(z, Z)=-4 \pi e^{2}\{\tilde{N}(z)-\tilde{N}(Z)\}
$$

Therefore it is conservative, as it depends on $t$ only through $z_{e}(t, Z)$ (resp on $\xi$ only through $\hat{z}_{e}(\xi, Z)$ ), and has the opposite sign with respect to the displacement $\Delta:=z-Z$ (like an elastic force); the associated potential energy is convex and with a minimum at $z=Z$ for every $Z$ and reads

$$
U(z ; Z)=4 \pi e^{2}[\tilde{\mathcal{N}}(z)-\tilde{\mathcal{N}}(Z)-\tilde{N}(Z)(z-Z)], \quad \tilde{\mathcal{N}}(Z):=\int_{0}^{Z} d \zeta \widetilde{N}(\zeta)=\int_{0}^{Z} d \zeta \widetilde{n_{0}}(\zeta)(Z-y) .
$$

Defining $U$ we have fixed the free additive constant so that $U(Z, Z) \equiv 0$, i.e. the minimum value is zero. It is remarkable that the collective effect of the ions and of the other electrons amounts to a conservative and spring-like longitudinal force. 
The Hamilton equations for the electron fluid amount to (8) and (25), where the latter now become

$$
\hat{s}_{e}^{\prime}=\frac{e}{m c^{2}}\left\{4 \pi e\left[\widetilde{N}\left(\hat{z}_{e}\right)-\widetilde{N}(Z)\right]+\left(\hat{\boldsymbol{x}}_{e}^{\perp \prime} \wedge \hat{\boldsymbol{B}}_{s}^{\perp}\right)^{z}\right\}, \quad \hat{\boldsymbol{u}}_{e}^{\perp \prime}=\frac{-e}{m c^{2}}\left[\left(\hat{\boldsymbol{x}}_{e}^{\prime} \wedge \hat{\boldsymbol{B}}_{s}\right)^{\perp}-\boldsymbol{\alpha}^{\perp \prime}\right] .
$$

We emphasize that they make up a family (parametrized by $Z$ ) of decoupled ODEs. As said, from (61) and causality it follows that $\boldsymbol{x}_{h}(t, \boldsymbol{X})=\boldsymbol{X}, \boldsymbol{u}_{e}(t, \boldsymbol{X})=\mathbf{0}$ if $\xi=c t-z \leq 0$, whence

$$
\hat{\boldsymbol{x}}_{e}(0, \boldsymbol{X})=\boldsymbol{X}, \quad \hat{\boldsymbol{u}}_{e}(0, \boldsymbol{X})=\mathbf{0} ;
$$

these can be adopted as the ( $\boldsymbol{X}$-parametrized family of) initial conditions for these ODEs.

Replacing the solution in the right-hand side of (68) one obtains a first correction to $\boldsymbol{A}^{\perp}$. The procedure can be iterated: replacing in (72) $\boldsymbol{\alpha}^{\perp}$ by the improved $\boldsymbol{A}^{\perp}$ one obtains an improved system of ODEs to determine the electrons motion, and so on.

As an illustration, we now briefly report some results of the numerical resolution, for small $Z, t$ and $\boldsymbol{B}_{s}=\mathbf{0}$, of the decoupled Cauchy problems (72-73). As in section 2.2.1, (72b) is solved by $\hat{\boldsymbol{u}}_{e}^{\perp}(\xi)=\boldsymbol{w}^{\perp}(\xi)$, and $\hat{v}=\hat{\boldsymbol{w}}^{\perp 2}$. The Hamiltonian and the Hamilton equations [in the unknowns $\left.\hat{z}_{e}(\xi, Z), \hat{s}(\xi, Z)\right]$ for the $Z$ electrons become [15]

$$
\begin{array}{r}
H\left(\hat{z}_{e}, \hat{s} ; \xi, Z\right) \equiv m c^{2} \gamma(\hat{s}, \xi)+U\left(\hat{z}_{e} ; Z\right), \quad \gamma(s, \xi) \equiv \frac{s^{2}+1+v(\xi)}{2 s}, \\
\hat{z}_{e}^{\prime}=\frac{1+\hat{v}}{2 \hat{s}^{2}}-\frac{1}{2}, \quad \hat{s}^{\prime}=\frac{4 \pi e^{2}}{m c^{2}}\left\{\tilde{N}\left[\hat{z}_{e}\right]-\tilde{N}(Z)\right\}
\end{array}
$$

$U(\cdot, Z)$ plays the role of $q A^{0}$ in (36). Once these equations are solved then (8a) is solved by quadrature as in (32). If in particular the initial density is constant, $\widetilde{n_{0}}(Z)=n_{0}$, then in terms of the displacement $\Delta:=z-Z$ (70-71) become $Z$-independent

$$
F_{e}^{z}(z, Z)=-4 \pi n_{0} e^{2} \Delta, \quad \mathcal{U}(\Delta ; Z)=2 \pi n_{0} e^{2} \Delta^{2},
$$

whence (75) reduce for all $Z$ to the same system of two first order ODEs

$$
\hat{\Delta}^{\prime}=\frac{1+\hat{v}}{2 \hat{s}^{2}}-\frac{1}{2}, \quad \hat{s}^{\prime}=M \hat{\Delta},
$$



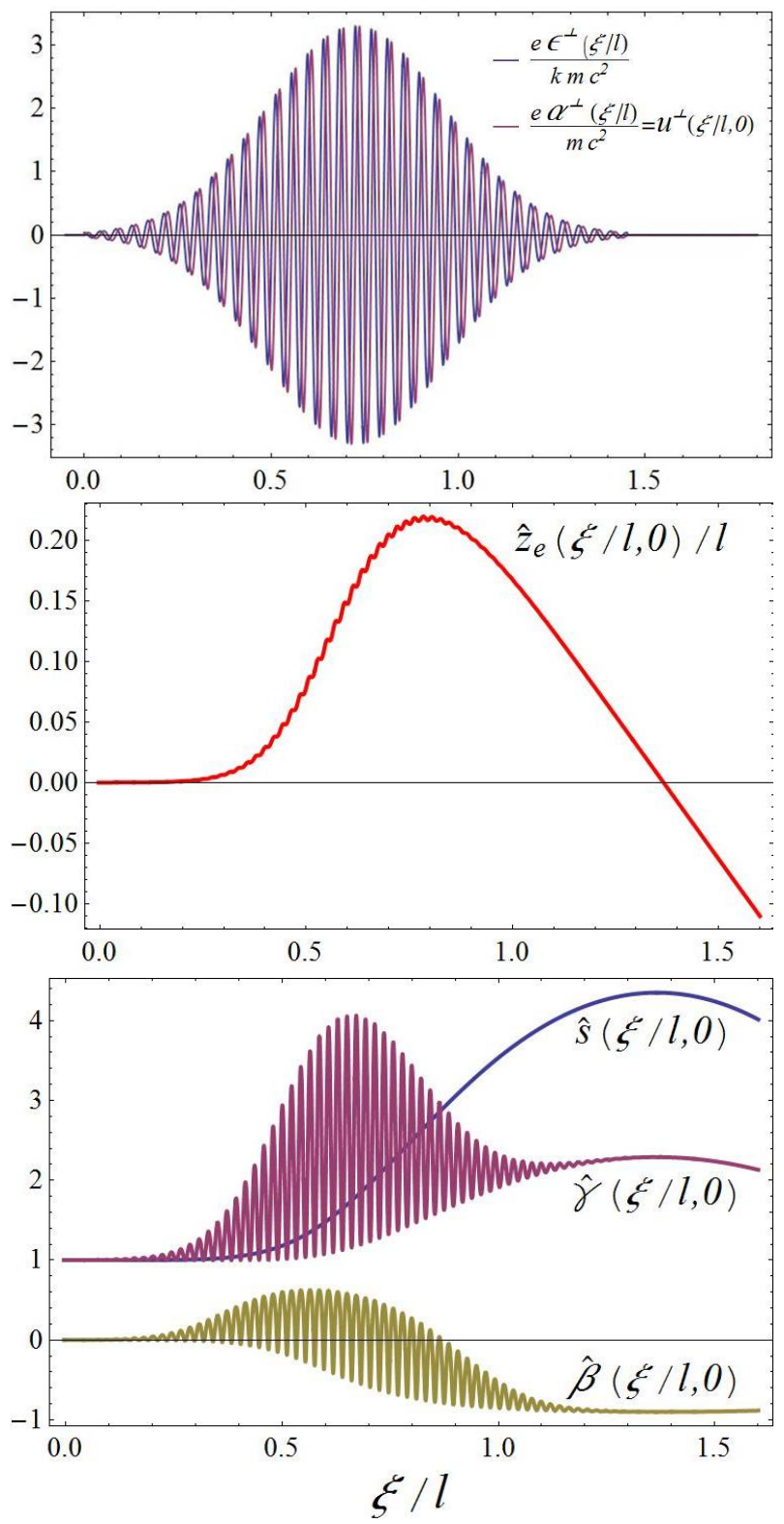

Figure 5: Normalized pump $\boldsymbol{\epsilon}^{\perp}$ as in fig. 2 right, $\boldsymbol{u}^{\perp}$ and solution of the electron equations (75) with $\boldsymbol{X}=\mathbf{0}$ and zero initial velocity in the interval from the beginning of the laser plasma interaction $(\xi=0)$ to shortly after the expulsion from the plasma bulk, assuming the initial density is $\widetilde{n_{0}}(Z)=n_{0} \theta(Z)$, with $n_{0}=21 \times 10^{17} \mathrm{~cm}^{-3}$.
$\left(\hat{\Delta}(\xi, Z) \quad:=\hat{z}_{e}(\xi, Z)-Z, \quad M \equiv\right.$ $4 \pi n_{0} e^{2} / m c^{2}=\omega_{p}^{2} / c^{2}$, where $\omega_{p}$ is the plasma frequency) with the same trivial intial conditions, $\hat{\Delta}(0, Z)=0, \hat{s}(0, Z)=1$; hence, every $Z$-layer of electrons behaves as an independent copy of the same relativistic harmonic oscillator ${ }^{a}$. If $\widetilde{n_{0}}(Z)=$ $n_{0} \theta(Z)$ (step-shaped initial density) then (76), (77b) hold only for $z \geq 0$, whereas for $z<0 F_{e}^{z}(z, Z)=4 \pi n_{0} e^{2} Z=$ const and $\hat{s}^{\prime}=-M Z$, as in the previous subsection. In fig. 5 we plot the $\boldsymbol{\epsilon}^{\perp}$ of a suitable ultra-short and ultra-intense laser pulse (the "pump") and the first part of the corresponding solution of (75) with zero initial velocity and $Z=0$ : tuning the electron density in the range where the plasma oscillation period is about twice the pulse duration, the $Z=0$ electrons are first boosted into the bulk by the positive part of the ponderomotive force $F_{p}^{z}$ due to the pulse, then are accelerated back by the negative force due to the charge displacements and the negative part of $F_{p}^{z}$. Note how smooth $\hat{s}(\xi)$ is, regardless of the fast and intense oscillations of $\boldsymbol{\epsilon}^{\perp}, \boldsymbol{\alpha}^{\perp}$; this is explained by remark 2.2.1.5. This motion is at the basis of the prediction of the slingshot effect, i.e. of the backward expulsion of high energy electrons just after a very short and intense laser pulse has hit the surface of a low density plasma $[34,15,16]$; the expelled electrons belong to the most superficial layer (smallest $Z$ ) of the plasma. The motion of the more internal electrons, leading to the formation of a plasma wave, will be studied in [43].

${ }^{a}$ When $\hat{v}=0$ then (77) implies $\hat{\Delta}^{\prime \prime}=-M \hat{\Delta} / \hat{s}^{3}$. In the nonrelativistic regime $\hat{s} \simeq 1$ and this becomes the nonrelativistic harmonic equation $\hat{\Delta}^{\prime \prime}=-\hat{\Delta} \omega_{p}^{2} / c^{2}$ with period $2 \pi / \omega_{p}$ in $t$. 


\section{Acknowledgments}

I am grateful to Renato Fedele and Sergio De Nicola for useful discussions. We acknowledge partial support by GNFM (Gruppo Nazionale di Fisica Matematica) of INdAM.

Note added in proof: A short summary about the present results can be found in [47].

\section{Appendix}

\subsection{Lagrange equations in the presence of additional forces}

If some additional force $\boldsymbol{Q}(\boldsymbol{x}, \dot{\boldsymbol{x}}, t)$ [e.g. a friction term $\boldsymbol{Q}=-\boldsymbol{\beta} f(\beta), f(\beta) \geq 0$ ] acts on the particle then the usual Lagrange equations of motions read

$$
\frac{d}{d t} \frac{\partial L}{\partial \dot{\boldsymbol{x}}}-\frac{\partial L}{\partial \boldsymbol{x}}=\boldsymbol{Q}
$$

Proposition 10 The Lagrange equations (78) are equivalent to the new ones

$$
\frac{d}{d \xi} \frac{\partial \mathcal{L}}{\partial \hat{\boldsymbol{x}}^{\prime}}-\frac{\partial \mathcal{L}}{\partial \hat{\boldsymbol{x}}}=\hat{\boldsymbol{Q}}
$$

where $\hat{\boldsymbol{Q}}^{\perp}\left(\hat{\boldsymbol{x}}, \hat{\boldsymbol{x}}^{\prime}, \xi\right):=\left(1+\hat{z}^{\prime}\right) \boldsymbol{Q}^{\perp}\left(\hat{\boldsymbol{x}}, \frac{c \hat{\boldsymbol{x}}^{\prime}}{1+\hat{z}^{\prime}} \frac{\xi+\hat{z}}{c}\right), \hat{Q}^{z}\left(\hat{\boldsymbol{x}}, \hat{\boldsymbol{x}}^{\prime}, \xi\right):=Q^{z}\left(\hat{\boldsymbol{x}}, \frac{c \hat{\boldsymbol{x}}^{\prime}}{1+\hat{z}^{\prime}}, \frac{\xi+\hat{z}}{c}\right)-\hat{\boldsymbol{x}}^{\perp \prime} \cdot \boldsymbol{Q}^{\perp}\left(\hat{\boldsymbol{x}}, \frac{c \hat{\boldsymbol{x}}^{\prime}}{1+\hat{z}^{\prime}}, \frac{\xi+\hat{z}}{c}\right)$.

Radiative losses could be accounted for by introducing in $\boldsymbol{Q}$ the Lorentz-Dirac term [44], which depends on higher $t$-derivatives of $\boldsymbol{x}$; correspondingly, $\hat{\boldsymbol{Q}}$ will depend also on on higher $\xi$-derivatives of $\hat{\boldsymbol{x}}$.

Proof: For any function $f(\boldsymbol{x}, \dot{\boldsymbol{x}}, t)$ we abbreviate $\left.f\right|_{\mathcal{R}}\left(\hat{\boldsymbol{x}}, \hat{\boldsymbol{x}}^{\prime}, \xi\right):=f\left[\hat{\boldsymbol{x}}, c \hat{\boldsymbol{x}}^{\prime} /\left(1+\hat{z}^{\prime}\right),(\xi+\hat{z}) / c\right]$. Using $d / d \xi=\left(1+\hat{z}^{\prime}\right) d / d(c t)$ and (78) we find

$$
\begin{aligned}
& \frac{\partial \mathcal{L}}{\partial \hat{\boldsymbol{x}}^{\perp \prime}}=\left.c \frac{\partial L}{\partial \dot{\boldsymbol{x}}^{\perp}}\right|_{\mathcal{R}}, \quad \frac{\partial \mathcal{L}}{\partial \hat{\boldsymbol{x}}^{\perp}}=\left.\left(1+\hat{z}^{\prime}\right) \frac{\partial L}{\partial \boldsymbol{x}^{\perp}}\right|_{\mathcal{R}}, \\
& \frac{d}{d \xi} \frac{\partial \mathcal{L}}{\partial \hat{\boldsymbol{x}}^{\perp \prime}}-\frac{\partial \mathcal{L}}{\partial \hat{\boldsymbol{x}}^{\perp}}=\left(1+\hat{z}^{\prime}\right)\left[\frac{d}{d t} \frac{\partial L}{\partial \dot{\boldsymbol{x}}^{\perp}}-\frac{\partial L}{\partial \boldsymbol{x}^{\perp}}\right]_{\mathcal{R}}=\left.\left(1+\hat{z}^{\prime}\right) \boldsymbol{Q}^{\perp}\right|_{\mathcal{R}}=: \hat{\boldsymbol{Q}}^{\perp}\left(\hat{\boldsymbol{x}}, \hat{\boldsymbol{x}}^{\prime}, \xi\right), \\
& \frac{\partial \mathcal{L}}{\partial \hat{z}}=\left(1+\hat{z}^{\prime}\right)\left[\frac{\partial L}{\partial z}+\frac{1}{c} \frac{\partial L}{\partial t}\right]_{\mathcal{R}}, \quad \frac{\partial \mathcal{L}}{\partial \hat{z}^{\prime}}=\left[L-\frac{c \hat{\boldsymbol{x}}^{\prime}}{1+\hat{z}^{\prime}} \cdot \frac{\partial L}{\partial \dot{\boldsymbol{x}}}+c \frac{\partial L}{\partial \dot{z}}\right]_{\mathcal{R}}=\left[L+c \frac{\partial L}{\partial \dot{z}}-\dot{\boldsymbol{x}} \cdot \frac{\partial L}{\partial \dot{\boldsymbol{x}}}\right]_{\mathcal{R}} \\
& \frac{d}{d \xi} \frac{\partial \mathcal{L}}{\partial \hat{z}^{\prime}}-\frac{\partial \mathcal{L}}{\partial \hat{z}}=\left(1+\hat{z}^{\prime}\right)\left\{\frac{d}{d t}\left[\frac{L}{c}+\frac{\partial L}{\partial \dot{z}}-\frac{\dot{\boldsymbol{x}}}{c} \cdot \frac{\partial L}{\partial \dot{\boldsymbol{x}}}\right]-\left[\frac{\partial L}{\partial z}+\frac{1}{c} \frac{\partial L}{\partial t}\right]\right\}_{\mathcal{R}} \\
& =\left(1+\hat{z}^{\prime}\right)\left\{Q^{z}+\left[\frac{d}{d t}-\frac{\partial}{\partial t}\right] \frac{L}{c}-\frac{d}{d t} \frac{\dot{\boldsymbol{x}}}{c} \cdot \frac{\partial L}{\partial \dot{\boldsymbol{x}}}\right\}_{\mathcal{R}}=\left(1+\hat{z}^{\prime}\right)\left\{Q^{z}+\frac{\dot{\boldsymbol{x}}}{c} \cdot \frac{\partial L}{\partial \boldsymbol{x}}+\frac{\ddot{\boldsymbol{x}}}{c} \cdot \frac{\partial L}{\partial \dot{\boldsymbol{x}}}-\left[\frac{\ddot{\boldsymbol{x}}}{c} \cdot \frac{\partial L}{\partial \dot{\boldsymbol{x}}}+\frac{\dot{\boldsymbol{x}}}{c} \cdot \frac{d}{d t} \frac{\partial L}{\partial \dot{\boldsymbol{x}}}\right]\right\}_{\mathcal{R}} \\
& =\left(1+\hat{z}^{\prime}\right)\left[Q^{z}-\frac{\dot{\boldsymbol{x}}}{c} \cdot \boldsymbol{Q}\right]_{\mathcal{R}}=\left(1+\hat{z}^{\prime}\right)\left[\left(1-\frac{\dot{z}}{c}\right) Q^{z}-\frac{\dot{\boldsymbol{x}}^{\perp}}{c} \cdot \boldsymbol{Q}^{\perp}\right]_{\mathcal{R}}=\left[Q^{z}-\hat{\boldsymbol{x}}^{\perp \prime} \cdot \boldsymbol{Q}^{\perp}\right]_{\mathcal{R}}=: \hat{Q}^{z}\left(\hat{\boldsymbol{x}}, \hat{\boldsymbol{x}}^{\prime}, \xi\right) .
\end{aligned}
$$




\subsection{Proof of Proposition 1 and of eq. (31)}

Proving that (13a) amount to (8) is straightforward. As for (13b), from the definition $\hat{f}(\xi, \hat{\boldsymbol{x}}):=f[(\xi+\hat{z}) / c, \hat{\boldsymbol{x}}]$ applied to $\hat{A}^{\mu}$ and its derivatives it follows

$$
\begin{aligned}
& \frac{d \hat{A}^{\mu}}{d \xi}=\frac{d}{d \xi} A^{\mu}\left[\frac{\xi+\hat{z}(\xi)}{c}, \hat{\boldsymbol{x}}(\xi)\right]=\frac{1+\hat{z}^{\prime}}{c} \widehat{\partial_{t} A^{\mu}}+\hat{x}^{i \prime} \widehat{\partial_{i} A^{\mu}}=\frac{1}{\hat{s}}\left[\frac{\hat{\gamma}}{c} \widehat{\partial_{t} A^{\mu}}+\hat{u}^{i} \widehat{\partial_{i} A^{\mu}}\right], \\
& \frac{\partial \hat{A}^{\mu}}{\partial \hat{z}}=\widehat{\partial_{z} A^{\mu}}+\frac{1}{c} \widehat{\partial_{t} A^{\mu}}, \quad \frac{\partial \hat{A}^{\mu}}{\partial \hat{x}^{a}}=\widehat{\partial_{a} A^{\mu}}, \quad a, b \in\{1,2\}, \quad i, j \in\{1,2,3\} .
\end{aligned}
$$

Setting $A^{-}=A^{0}-A^{z}$ and using the relations between $A^{\mu}$ and $\boldsymbol{E}, \boldsymbol{B}$ as well as (7) we find

$$
\begin{aligned}
& 0=\hat{\Pi}^{a \prime}+\frac{\partial \hat{H}}{\partial \hat{x}^{a}}=m c^{2} \hat{u}^{a \prime}+q \frac{d \hat{A}^{a}}{d \xi}+q \frac{\partial \hat{A}^{0}}{\partial \hat{x}^{a}}-q \frac{\hat{u}^{b}}{\hat{s}} \frac{\partial \hat{A}^{b}}{\partial \hat{x}^{a}}+\frac{q}{2}\left[\frac{1+\hat{\boldsymbol{u}}^{\perp 2}}{\hat{s}^{2}}-1\right] \frac{\partial \hat{A}^{-}}{\partial \hat{x}^{a}} \\
& =m c^{2} \hat{u}^{a \prime}+\frac{q}{\hat{s}}\left[\hat{u}^{i} \widehat{\partial_{i} A^{a}}+\frac{\hat{\gamma}}{c} \widehat{\partial_{t} A^{a}}\right]+q \widehat{\partial_{a} A^{0}}-q \frac{\hat{u}^{b}}{\hat{s}} \widehat{\partial_{a} A^{b}}+q \frac{\hat{u}^{z}}{\hat{s}}\left(\widehat{\partial_{a} A^{0}}-\widehat{\partial_{a} A^{z}}\right) \\
& =m c^{2} \hat{u}^{a \prime}+\frac{q}{\hat{s}} \hat{u}^{b}\left(\widehat{\partial_{b} A^{a}}-\widehat{\partial_{a} A^{b}}\right)+\frac{q}{\hat{s}} \hat{u}^{z}\left(\widehat{\partial_{z} A^{a}}-\widehat{\partial_{a} A^{z}}\right)+\frac{q \hat{\gamma}}{c \hat{s}} \widehat{\partial_{t} A^{a}}+q\left(1+\frac{\hat{u}^{z}}{\hat{s}}\right) \widehat{\partial_{a} A^{0}} \\
& =m c^{2} \hat{u}^{a \prime}-\frac{q}{\hat{s}} \varepsilon^{a b z} \hat{u}^{b} \hat{B}^{z}-\frac{q}{\hat{s}} \varepsilon^{a z b} \hat{u}^{z} \hat{B}^{b}+\frac{q \hat{\gamma}}{\hat{s}}\left(\frac{1}{c} \widehat{\partial_{t} A^{a}}+\widehat{\partial_{a} A^{0}}\right) \\
& =m c^{2} \hat{u}^{a \prime}-\frac{q}{\hat{s}} \varepsilon^{a i j} \hat{u}^{i} \hat{B}^{j}-\frac{q \hat{\gamma}}{\hat{s}} \hat{E}^{a}=m c^{2} \hat{u}^{a \prime}-\frac{q}{\hat{s}}[\hat{\boldsymbol{u}} \wedge \hat{\boldsymbol{B}}+\hat{\gamma} \hat{\boldsymbol{E}}]^{a}, \quad a=x, y, \\
& 0=\hat{\Pi}^{z \prime}+\frac{\partial \hat{H}}{\partial \hat{z}}=-m c^{2} \hat{s}^{\prime}-q \frac{d \hat{A}^{-}}{d \xi}+q \frac{\partial \hat{A}^{0}}{\partial \hat{z}}-q \frac{\hat{u}^{a}}{\hat{s}} \frac{\partial \hat{A}^{a}}{\partial \hat{z}}+q \frac{\hat{u}^{z}}{\hat{s}} \frac{\partial \hat{A}^{-}}{\partial \hat{z}} \\
& =-m c^{2} \hat{s}^{\prime}-\frac{q}{\hat{s}}\left[\frac{\hat{\gamma}}{c} \widehat{\partial_{t} A^{-}}+\hat{u}^{i} \widehat{\partial_{i} A^{-}}\right]+q \frac{\hat{u}^{z}}{\hat{s}}\left(\widehat{\partial_{z} A^{-}}+\frac{1}{c} \widehat{\partial_{t} A^{-}}\right)+q \frac{\partial \hat{A}^{0}}{\partial \hat{z}}-q \frac{\hat{u}^{a}}{\hat{s}} \frac{\partial \hat{A}^{a}}{\partial \hat{z}} \\
& =-m c^{2} \hat{s}^{\prime}-\frac{q}{\hat{s}}\left[\frac{\hat{\gamma}-\hat{u}^{z}}{c} \widehat{\partial_{t} A^{-}}+\hat{u}^{a} \widehat{\partial_{a} A^{-}}\right]+q \frac{\partial \hat{A}^{0}}{\partial \hat{z}}-q \frac{\hat{u}^{a}}{\hat{s}} \frac{\partial \hat{A}^{a}}{\partial \hat{z}} \\
& =-m c^{2} \hat{s}^{\prime}-\frac{q}{c}\left(\widehat{\partial_{t} A^{0}}-\widehat{\partial_{t} A^{z}}\right)-\frac{q}{\hat{s}} \hat{u}^{a}\left(\widehat{\partial_{a} A^{0}}-\widehat{\partial_{a} A^{z}}\right)+q\left(\widehat{\partial_{z} A^{0}}+\frac{\widehat{\partial_{t} A^{0}}}{c}\right)-q \frac{\hat{u}^{a}}{\hat{s}}\left(\widehat{\partial_{z} A^{a}}+\frac{\widehat{\partial_{t} A^{a}}}{c}\right) \\
& =-m c^{2} \hat{s}^{\prime}+q\left(\frac{1}{c} \widehat{\partial_{t} A^{z}}+\widehat{\partial_{z} A^{0}}\right)-\frac{q}{\hat{s}} \hat{u}^{a}\left(\widehat{\partial_{a} A^{0}}+\frac{1}{c} \widehat{\partial_{t} A^{a}}\right)+q \frac{\hat{u}^{a}}{\hat{s}}\left(\widehat{\partial_{a} A^{z}}-\widehat{\partial_{z} A^{a}}\right) \\
& =-m c^{2} \hat{s}^{\prime}-q \hat{E}^{z}+\frac{q}{\hat{s}} \hat{u}^{a} \hat{E}^{a}-\frac{q}{\hat{s}} \varepsilon^{z a b} \hat{u}^{a} \hat{B}^{b}=-m c^{2} \hat{s}^{\prime}-q \hat{E}^{z}+\frac{q}{\hat{s}}\left[\hat{\boldsymbol{u}}^{\perp} \cdot \hat{\boldsymbol{E}}^{\perp}-\left(\hat{\boldsymbol{u}}^{\perp} \wedge \hat{\boldsymbol{B}}^{\perp}\right)^{z}\right],
\end{aligned}
$$

as claimed. (14) can be obtained also directly from (11b), using the relation $d / d t=$ $(c \hat{s} / \hat{\gamma}) d / d \xi$. Eq. (15) is obtained as usual from $d \hat{H} / d \xi=\left(\partial \hat{H} / \partial \hat{x}^{i}\right) \hat{x}^{i \prime}+\left(\partial \hat{H} / \partial \hat{\Pi}^{i}\right) \hat{\Pi}^{i \prime}+\partial \hat{H} / \partial \xi$ and (13).

If $A^{\mu}=A^{\mu}(t, z)$, then $\partial_{a} A^{\mu}=0$, and from (81), (30) it follows (31), as claimed:

$$
\begin{aligned}
0 & =-m c^{2} \hat{s}^{\prime}-q \hat{E}^{z}-\frac{q}{\hat{s}} \hat{u}^{a}\left(\frac{1}{c} \widehat{\partial_{t} A^{a}}+\widehat{\partial_{z} A^{a}}\right)=-m c^{2} \hat{s}^{\prime}-q \hat{E}^{z}-\frac{q}{\hat{s}} \hat{u}^{a} \frac{\partial \hat{A}^{a}}{\partial \hat{z}} \\
& =-m c^{2} \hat{s}^{\prime}-q \hat{E}^{z}+\frac{m c^{2}}{2 \hat{s}} \frac{\partial \hat{\boldsymbol{u}}^{\perp 2}}{\partial \hat{z}} .
\end{aligned}
$$




\subsection{Generalized canonical transformations}

Given a Hamiltonian system, a generalized canonical (or contact) transformation can be defined as a transformation of coordinates $(Q, P, t) \mapsto(\Theta, \Pi, T)$ in extended phase space which preserves the Hamiltonian form of the equations of motion. Since the latter can be formally derived from Hamilton's principle - written in the form $\delta S=\delta \int\left(\sum_{i} P_{i} d Q^{i}-H d t\right)=$ 0 - by varying $Q, P$ independently (see e.g. [46], p. 140), there must exist a function $F$ such that

$$
d F=\sum_{i} P_{i} d Q^{i}-H d t-\left(\sum_{i} \Pi_{i} d \Theta^{i}-K d T\right),
$$

so that the old and the new actions differ only by a constant (the difference of $F$ at the integration endpoints), which does not contribute to the variation. Here $T, K$ stand for the new "time" and Hamiltonian, respectively; $d T / d t$ must be positive-definite. If $T=t$ we obtain the usual formula, eq. (45.6) in [46]. If $(Q, \Theta, t)$ are a set of coordinates in the extended phase space we name the transformation as free with (first-type) generating function $F(Q, \Theta, t)$, and $P, \Pi, H$ are determined by

$$
P_{i}=\frac{\partial F}{\partial Q^{i}}-K \frac{\partial T}{\partial Q^{i}}, \quad \Pi_{i}=-\frac{\partial F}{\partial \Theta^{i}}+K \frac{\partial T}{\partial \Theta^{i}}, \quad H=K \frac{\partial T}{\partial t}-\frac{\partial F}{\partial t} .
$$

As in the usual setting, the identical transformation is not free. Eq. (82) is equivalent to

$$
d\left(F+\sum_{i} \Pi_{i} \Theta^{i}\right)=\sum_{i}\left(P_{i} d Q^{i}+\Theta^{i} d \Pi_{i}\right)-H d t+K d T ;
$$

if $(Q, \Pi, t)$ are a set of coordinates in the extended phase space, we can express the argument of the left differential as a function $\Phi(Q, \Pi, t)$, and $P, \Theta, H$ are determined by

$$
P_{i}=\frac{\partial \Phi}{\partial Q^{i}}-K \frac{\partial T}{\partial Q^{i}}, \quad \Theta^{i}=\frac{\partial \Phi}{\partial \Pi^{i}}-K \frac{\partial T}{\partial \Pi^{i}}, \quad H=K \frac{\partial T}{\partial t}-\frac{\partial \Phi}{\partial t} .
$$

We name $\Phi$ the second-type generating function of the transformation. The identical one has generating function $\Phi=\sum_{i} \Pi_{i} Q^{i}$. As in the usual theory, also generating functions depending on different sets of old and new coordinates can be introduced; each of the latter needs to be a set of coordinates in extended phase space. If $T=t$ we obtain the usual formulae ${ }^{9}$. Identifying $Q^{i} \equiv x^{i}(i=1,2,3)$ and $T \equiv \xi / c$, the transformation introduced in section $2(\boldsymbol{x}, \boldsymbol{P}, t) \mapsto(\boldsymbol{x}, \boldsymbol{\Pi} / c, T)$, with

$$
\boldsymbol{P}^{\perp}=\Pi^{\perp}, \quad P^{z}=\Pi^{z}+H, \quad \Theta^{i}=x^{i}, \quad K=H
$$

(here we have removed the caret, which is only added to distinguish the dependence of a dynamical variable on $\xi$ rather than on $t$ ), by construction is generalized canonical with $F \equiv 0$ [because the action (4) in terms of the old and new variables is the same]; it is generated by $\Phi=\sum_{i} \Pi_{i} x^{i}$.

${ }^{9}$ Comparing our results e.g. with section 45 of [46] we find that our (84) yields (45.8) of [46],

$$
P_{i}=\frac{\partial \Phi}{\partial Q^{i}}, \quad \Theta^{i}=\frac{\partial \Phi}{\partial \Pi^{i}}, \quad K=H+\frac{\partial \Phi}{\partial t} .
$$


We recall that for fixed initial position $Q_{0}$ at time $t_{0}$ the action function $S(Q, t)$ is defined as the value of the action functional $S(\lambda)$ along the worldline $\lambda_{e}$ connecting $\left(Q_{0}, t_{0}\right)$ with $(Q, t)$ and fulfilling $\left.\delta S\right|_{\lambda_{e}}=0 ; S(Q, t)$ fulfills the Hamilton-Jacobi equation

$$
-\frac{\partial S}{\partial t}=H\left(Q, \frac{\partial S}{\partial Q}, t\right)
$$

For the problem considered here $H(\boldsymbol{x}, \boldsymbol{P}, t)=\sqrt{m^{2} c^{4}+(c \boldsymbol{P}-q \boldsymbol{A})^{2}}+q A^{0}$. Choosing $A(x)=$ $\boldsymbol{\alpha}^{\perp}(c t-z) \cdot d \boldsymbol{x}^{\perp}$ as in subsection 3.1 and taking the square, the equation for $S(\boldsymbol{x}, t)$ becomes

$$
\left(\frac{\partial S}{\partial t}\right)^{2}=m^{2} c^{4}+\left[c \nabla S-q \boldsymbol{\alpha}^{\perp}(c t-z)\right]^{2}
$$

The function

$$
\Phi(\boldsymbol{x}, t ; \boldsymbol{\Pi})=\frac{\boldsymbol{\Pi}}{c} \cdot \boldsymbol{x}+\frac{1}{2 c \Pi^{z}} \int_{\xi_{0}}^{c t-z} d \zeta\left\{m^{2} c^{4}+\left[\boldsymbol{\Pi}-q \boldsymbol{\alpha}^{\perp}(\zeta)\right]^{2}\right\}=\frac{\boldsymbol{\Pi}}{c} \cdot \boldsymbol{x}-\int_{\xi_{0}}^{c t-z} \frac{d \zeta}{c} \hat{H}\left[\boldsymbol{x}^{\perp}, \zeta, \boldsymbol{\Pi}\right]
$$

( $\hat{H}$ depends on $\zeta$ through the argument of $\boldsymbol{\alpha}^{\perp}$ ) is a complete integral of (85), i.e. a solution depending on three additional constants $\Pi^{i}$. We can interpret them as the conjugate variables of the $x^{i}$, since in subsection 3.1 we have shown that the latter are constant. According to general principles, also the $\Theta^{i}=c \partial \Phi / \partial \Pi^{i}$ must be constant. Replacing (38) in (9) we find by a straightforward calculation that in fact these are the initial conditions: $\Theta^{i}=x_{0}^{i}$. We find the same result more directly using the Hamilton equations:

$$
\Theta^{i}=c \frac{\partial \Phi}{\partial \Pi^{i}}=\hat{x}^{i}(\xi)-\int_{\xi_{0}}^{c t-z} d \zeta \frac{\partial \hat{H}}{\partial \Pi^{i}}\left[\boldsymbol{x}^{\perp}(\zeta), \zeta, \boldsymbol{\Pi}\right]=\hat{x}^{i}(\xi)-\int_{\xi_{0}}^{\xi} d \zeta \hat{x}^{i \prime}(\zeta)=\hat{x}^{i}\left(\xi_{0}\right)=x_{0}^{i} .
$$

Summing up, (86) is the generating function of the generalized canonical transformation $(\boldsymbol{x}, \boldsymbol{P}, t) \mapsto\left(\boldsymbol{x}_{0}, \boldsymbol{\Pi} / c, \xi / c\right)$. Up to the notation, it coincides with the one introduced at page 128 of [4].

\subsection{Estimates of oscillatory integrals}

Given $f \in \mathcal{S}(\mathbb{R})$ (the Schwartz space), integrating by parts we find for all $n \in \mathbb{N}$

$$
\begin{aligned}
\int_{-\infty}^{\xi} d \zeta f(\zeta) e^{i k \zeta} & =-\frac{i}{k} f(\xi) e^{i k \xi}+R_{1}^{f}(\xi) \\
=\ldots & =-\sum_{h=0}^{n-1}\left(\frac{i}{k}\right)^{h+1} f^{(h)}(\xi) e^{i k \xi}+R_{n}^{f}(\xi)
\end{aligned}
$$

where

$$
\begin{aligned}
& R_{1}^{f}(\xi):=\frac{i}{k} \int_{-\infty}^{\xi} d \zeta f^{\prime}(\zeta) e^{i k \zeta}=\left(\frac{i}{k}\right)^{2}\left[f^{\prime}(\xi) e^{i k \xi}+\int_{-\infty}^{\xi} d \zeta f^{\prime \prime}(\zeta) e^{i k \zeta}\right] \\
& R_{n}^{f}(\xi):=\left(\frac{i}{k}\right)^{n} \int_{-\infty}^{\xi} d \zeta f^{(n)}(\zeta) e^{i k \zeta}=\left(\frac{i}{k}\right)^{n+1}\left[f^{(n)}(\xi) e^{i k \xi}+\int_{-\infty}^{\xi} d \zeta f^{(n+1)}(\zeta) e^{i k \zeta}\right]
\end{aligned}
$$


Hence we find the following upper bounds for the remainders $R_{1}^{f}$, and more generally $R_{n}^{f}$ :

$$
\begin{aligned}
& \left|R_{1}^{f}(\xi)\right| \leq \frac{1}{|k|^{2}}\left[\left|f^{\prime}(\xi)\right|+\int_{-\infty}^{\xi} d \zeta\left|f^{\prime \prime}(\zeta)\right|\right] \leq \frac{\left\|f^{\prime}\right\|_{\infty}+\left\|f^{\prime \prime}\right\|_{1}}{|k|^{2}}, \\
& \left|R_{n}^{f}(\xi)\right| \leq \frac{1}{|k|^{n+1}}\left[f^{(n)}(\xi)+\int_{-\infty}^{\xi} d \zeta\left|f^{(n+1)}(\zeta)\right|\right] \leq \frac{\left\|f^{(n)}\right\|_{\infty}+\left\|f^{(n+1)}\right\|_{1}}{|k|^{n+1}} .
\end{aligned}
$$

It follows $R_{1}^{f}=O\left(1 / k^{2}\right)$, and more generally $R_{n}^{f}=O\left(1 / k^{n+1}\right)$, so that (88) are asymptotic expansions in 1/k. All inequalities in (90-91) are useful: the left inequalities are more stringent, while the right ones are $\xi$-independent.

Equations (87), (90) and $R_{1}^{f}=O\left(1 / k^{2}\right)$ hold also if $f \in W^{2,1}(\mathbb{R})$ (a Sobolev space), in particular if $f \in C^{2}(\mathbb{R})$ and $f, f^{\prime}, f^{\prime \prime} \in L^{1}(\mathbb{R})$, because the previous steps can be done also under such assumptions. Equations (87) will hold with a remainder $R_{1}^{f}=O\left(1 / k^{2}\right)$ also under weaker assumptions, e.g. if $f^{\prime}$ is bounded and piecewise continuous and $f, f^{\prime}, f^{\prime \prime} \in$ $L^{1}(\mathbb{R})$, although $R_{1}^{f}$ will be a sum of contributions like (89) for every interval in which $f^{\prime}$ is continuous. Similarly, (88), (91) and/or $R_{n}^{f}=O\left(1 / k^{n+1}\right)$ hold also under analogous weaker conditions.

Letting $\xi \rightarrow \infty$ in $(87)$, (90) we find for the Fourier transform $\tilde{f}(k):=\int_{-\infty}^{\infty} d \zeta f(\zeta) e^{-i k y}$ of $f(\xi)$

$$
|\tilde{f}(k)| \leq \frac{\left\|f^{\prime}\right\|_{\infty}+\left\|f^{\prime \prime}\right\|_{1}}{|k|^{2}}
$$

hence $\tilde{f}(k)=O\left(1 / k^{2}\right)$ as well. Actually, for functions $f \in \mathcal{S}(\mathbb{R})$ the decay of $\tilde{f}(k)$ as $|k| \rightarrow \infty$ is much faster, since $\tilde{f} \in \mathcal{S}(\mathbb{R})$ as well. For the gaussian $f(\xi)=\exp \left[-\xi^{2} / 2 \sigma\right]$ it is $\tilde{f}(k)=\sqrt{\pi \sigma} \exp \left[-k^{2} \sigma / 2\right]$.

To prove approximation (23) now we just need to choose $f=\epsilon$ and note that every component of $\boldsymbol{\alpha}^{\perp}$ will be a combination of (88) and $(88)_{k \mapsto-k}$.

\subsection{Cyclotron autoresonance}

Under the assumption of a slowly modulated monochromatic pulse (21-22), (19a) we can tune $\boldsymbol{B}_{s}=B^{z} \mathbf{k}$ so that the acceleration by the pulse becomes resonant (cyclotron autoresonance). We can obtain a straightforward good estimate applying approximation (23). We consider first the case of circular polarization: $w^{x}(\xi)+i w^{y}(\xi) \simeq e^{i k \xi} \mathrm{w}(\xi)$, where $\mathrm{w}(\xi):=q \epsilon(\xi) / k m c^{2}$ (normalized modulating amplitude; it is dimensionless). Hence eq. (47a) becomes

$$
(\hat{x}+i \hat{y})(\xi) \simeq i e^{-i b \xi} \int_{0}^{\xi} d \zeta \mathrm{w}(\zeta) e^{i(b+k) \zeta}
$$

If $b \neq-k$ then

$$
(\hat{x}+i \hat{y})(\xi) \simeq \frac{\mathrm{w}(\xi) e^{i k \xi}}{b+k}
$$


hence $\hat{\boldsymbol{x}}^{\perp}$, as well as $\hat{\boldsymbol{u}}^{\perp} \simeq \boldsymbol{w}^{\perp}|k| /|b+k|, \hat{v} \sim \mathrm{w}^{2} k^{2} /(b+k)^{2}$, leading to small accelerations. On the contrary, $b=-k$ leads to

$$
\begin{aligned}
& (\hat{x}+i \hat{y})(\xi) \simeq i e^{i k \xi} W(\xi), \quad\left(\hat{u}^{x}+i \hat{u}^{y}\right)(\xi) \simeq e^{i k \xi}[i \mathrm{w}(\xi)-k W(\xi)] \simeq-k e^{i k \xi} W(\xi), \\
& \hat{z}^{\prime}=\frac{\hat{\boldsymbol{u}}^{\perp 2}}{2} \simeq \frac{k^{2}}{2} W^{2}, \quad \hat{z}(\xi) \simeq \frac{k^{2}}{2} \int_{0}^{\xi} d \zeta W^{2}(\zeta) \quad \text { where } W(\xi):=\int_{0}^{\xi} d \zeta \mathrm{w}(\zeta)>0
\end{aligned}
$$

and therefore to a large longitudinal acceleration, because $W(\xi)$ increases monotonically. This is the so-called cyclotron autoresonance found in Ref. [23, 24] (see also [45]). In particular if $\boldsymbol{\epsilon}^{\perp}(\xi)=\mathbf{0}$ for $\xi \geq l \equiv$ pulse length then for such $\xi$

$$
\hat{u}^{z}(\xi)=\hat{z}^{\prime}(\xi) \simeq \frac{k^{2}}{2} W^{2}(l), \quad \frac{\left|\hat{\boldsymbol{x}}^{\perp \prime}(\xi)\right|}{\hat{z}^{\prime}(\xi)} \simeq \frac{2}{k W(l)} \ll 1 ;
$$

the final collimation is very good by the second formula. The final energy gain and the longitudinal displacement at the end of the interaction are

$$
\mathcal{E}_{f}=\frac{\hat{v}(l)}{2} \simeq \frac{k^{2}}{4} W^{2}(l), \quad(\Delta z)_{f} \simeq \frac{k^{2}}{2} \int_{0}^{l} d \zeta W^{2}(\zeta)
$$

The effect leads to remarkable accelerations if the amplitude $\epsilon$ of the pump is large, as it can be produced by modern lasers. In reality, no laser pulse can be considered as a plane wave, because it has a finite spot radius (i.e. transverse size); moreover, the latter is not constant along the path, and therefore the amplitude w cannot be considered as a function of $\xi$ only. As known, if at $\boldsymbol{x}=\mathbf{0}$ (say) the pulse has minimum spot radius $R$ (i.e. maximal focalization), for $z>0$ the spot radius increases monotonically with $z$ and is $\sqrt{2} R$ at $\boldsymbol{x}=z_{R} \mathbf{k}$, where $z_{R}=k R^{2} / 2$ is the Rayleigh length. Eq. (95) and (97) are reliable only if

$$
l \gg \lambda=\frac{2 \pi}{k}, \quad(\Delta z)_{f} \lesssim z_{R}=\frac{k}{2} R^{2}, \quad W(l) \simeq\left|\hat{\boldsymbol{x}}^{\perp}(l)\right| \lesssim R .
$$

The first is a condition for slow modulation, the second guarantees that during the pulseparticle interaction we can consider the spot radius as approximately constant (and equal to $R$ ), so that the normalized amplitude w can be approximated as a function of $\xi$ only, while the third guarantees that during the whole interaction the EM wave "seen" by the particle can be approximated as a plane travelling wave. Then the pulse EM energy is approximately

$$
\mathrm{E}=\int d V \frac{\boldsymbol{E}_{t}^{\perp 2}+\boldsymbol{B}_{t}^{\perp 2}}{8 \pi} \simeq \frac{R^{2}}{4} \int_{0}^{l} d \xi \epsilon_{s}^{2}(\xi)=\left[\frac{m c^{2} k R}{2 q}\right]^{2} \int_{0}^{l} d \xi \mathrm{w}^{2}(\xi)
$$

The main limitation of the above acceleration mechanism is that magnetic fields above $10^{5}$ Gauss are hardly achievable. Setting $B^{z}=10^{5} \mathrm{G}$ we find $k=b \simeq 60 \mathrm{~cm}^{-1}$ (i.e. $\lambda \simeq 1 \mathrm{~mm}$ ) if the charge particle is the electron (laser pulses with such carrier wave number can be produced e.g. by free electron lasers). We can obtain the order of magnitude of the effect by assuming the rough, simplifying Ansatz $\mathrm{w}(\xi)=\mathrm{w}_{0} \theta(\xi) \theta(l-\xi)$, whence

$$
W(l)=\mathrm{w}_{0} l, \quad(\Delta z)_{f} \simeq \frac{\left(k \mathrm{w}_{0}\right)^{2} l^{3}}{6}, \quad \mathrm{E} \simeq l\left[\frac{m c^{2} k \mathrm{w}_{0} R}{2 q}\right]^{2}, \quad \mathcal{E}_{f} \simeq \frac{\left(k \mathrm{w}_{0} l\right)^{2}}{4}
$$


Eq. (98) is fulfilled only if $R \gtrsim \mathrm{w}_{0} l \sqrt{k l / 3}$. We tune $R=\mathrm{w}_{0} l \sqrt{k l / 3}$ to obtain the maximum amplitude; correspondingly, $\mathrm{E} \simeq\left(m c^{2} \mathrm{w}_{0}^{2} l^{2}\right)^{2} k^{3} / 12 q^{2}$. In terms of $\mathrm{E}, k$ we find $\mathrm{w}_{0} l$ and

$$
R \simeq\left[\frac{4 q^{2} \mathrm{E} l^{2}}{3 m^{2} c^{4} k}\right]^{1 / 4}, \quad \mathcal{E}_{f} \simeq \frac{|q| \sqrt{3 k \mathrm{E}}}{m c^{2}}, \quad(\Delta z)_{f} \simeq \mathcal{E}_{f} \frac{2 l}{3} .
$$

For electrons $\mathcal{E}_{f}$ exceeds 1 , and therefore electrons become relativistic, when $\mathrm{E}$ exceeds $1.5 \times 10^{-3} \mathrm{~J} . \mathrm{E}=5 \mathrm{~J}$ gives $\mathcal{E}_{f}=\gamma_{f}-1 \simeq 28.5$ corresponding to electrons with a final energy of about $14.5 \mathrm{MeV}$ (independently of $l$ ). Choosing $l=2 \mathrm{~cm}$, we find $R \simeq 1 \mathrm{~cm},(\Delta z)_{f} \simeq 37 \mathrm{~cm}$ as the length of the accelerator, a result better than, but comparable with, the results achievable with traditional radio-frequency based accelerators (the latter typically produce an energy increase of $10 \mathrm{MeV}$ per meter). The corresponding approximate electron solution (95) is depicted in fig. 6. A higher $\mathcal{E}_{f}$ requires higher $\mathrm{E}$ or $B^{z}$. Of course, the energy gain for protons or other ions is much lower with presently available pulse energies, due to their much larger masses.
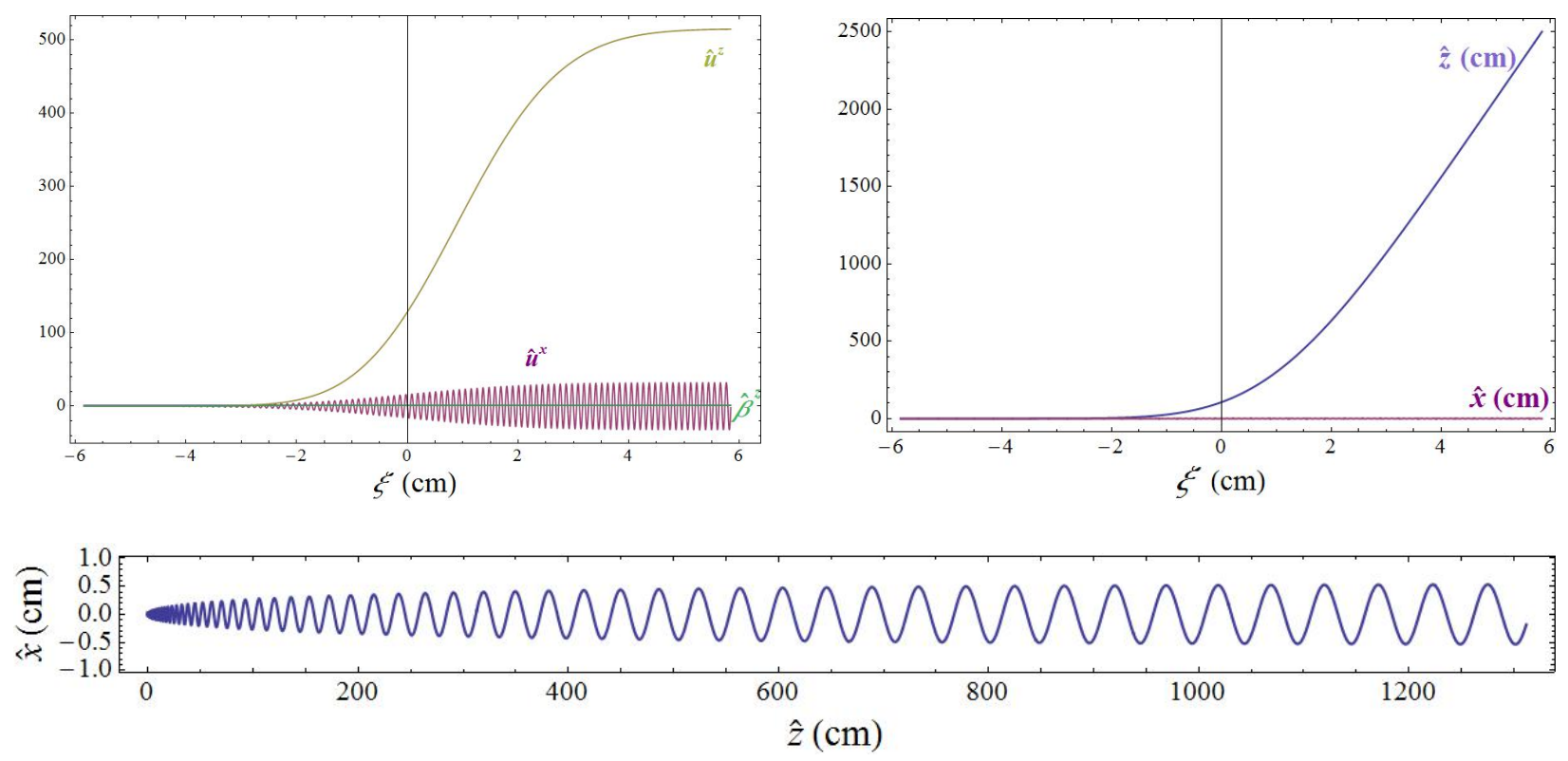

Figure 6: The approximate electron solution (95) (and the $z x$-projection of the corresponding trajectory) induced in a longitudinal magnetic field $B^{z}=10^{5} \mathrm{G}$ by a circularly polarized modulated pump (21-22) with wavelength $\lambda \simeq 1 \mathrm{~mm}, b=k \simeq 58.6 \mathrm{~cm}^{-1}$, gaussian enveloping amplitude $\epsilon(\xi)=a \exp \left[-\xi^{2} / 2 \sigma\right]$ with $\sigma=3 \mathrm{~cm}^{2}$ and $e a / k m c^{2}=0.15$, trivial initial conditions $\left(\boldsymbol{x}_{0}=\boldsymbol{\beta}_{0}=0\right)$, giving $\mathcal{E}_{f} \simeq 28.5$.

The results are completely analogous if the polarization of the pump is linear.

For strictly monochromatic waves $\epsilon \equiv$ const and all $\simeq$ above clearly become strict equalities. Up to our knowledge, $[23,24,45]$ and the rest of the literature have determined the solution of the equations of motion [in the form (94)] and proved the autoresonance for all $k=-b$ only in such a case. 


\section{References}

[1] J. D. Jackson, Classical electrodynamics, 3rd Edition, Wiley, 2007.

[2] W. K. H. Panofsky, M. Phillips, Classical Electricity and Magnetism, 2nd Edition, Courier Corporation, 2012.

[3] F. F. Chen, Introduction to plasma physics, Plenum Press, 1974.

[4] L.D. Landau, E.M. Lifshitz, Teoriya polya, $2^{\text {nd }}$ edition, Fizmatgiz, 1960; translated from the Russian in: The Classical Theory of Fields, $2^{\text {nd }}$ edition, Pergamon Press, 1962.

[5] D. Strickland, G. Mourou, Opt. Commun. 56 (1985), 219.

[6] M. D. Perry, G. Mourou, Science 264 (1994), 917; and references therein.

[7] G. A. Mourou, T. S. Tajima, V. Bulanov, Rev. Mod. Phys. 78 (2006), 309.

[8] G. Mourou, S. Mironov, E. Khazanov, A. Sergeev, Eur. Phys. J. ST 223 (2014), 1181.

[9] T. Tajima, K. Nakajima, G. Mourou, Riv. N. Cim. 40 (2017),34.

[10] T. Tajima, J.M. Dawson, Phys.Rev.Lett. 43, 267 (1979).

[11] L.M. Gorbunov, V.I. Kirsanov, Sov. Phys. JETP 66 (1987), 290.

[12] P. Sprangle, E. Esarey, A. Ting, G. Joyce, Appl. Phys. Lett. 53, 2146 (1988).

[13] E. Esarey, C. B. Schroeder, W. P. Leemans, Rev. Mod. Phys. 81 (2009), 1229.

[14] G. Fiore, J. Phys. A: Math. Theor. 47 (2014), 225501.

[15] G. Fiore, S. De Nicola, Phys Rev. Acc. Beams 19 (2016), 071302 (15pp).

[16] G. Fiore, S. De Nicola, Nucl. Instr. Meth. Phys. Res. A 829 (2016), 104-108.

[17] G. Fiore, Ricerche Mat. 65 (2016), 491-503.

[18] P. A. M. Dirac, Rev. Mod. Phys. 21 (1949), 392-399.

[19] R. A. Neville, F. Rohrlich, Phys. Rev. D 3 (1971), 1692.

[20] A. Di Piazza, Lett. Math. Phys. 83 (2008), 305-313.

[21] Y. Kravets, A. Noble, D. Jaroszynski, Phys. Rev. E 88 (2013), 011201(R).

[22] T. Heinzl, A. Ilderton, J. Phys. A 50 (2017), 345204; Phys. Rev. Lett. 118 (2017), 113202.

[23] A.A. Kolomenskii, A.N. Lebedev, Sov. Phys. Dokl. 7745 (1963) [Dokl. Akad. Nauk SSSR 1451259 (1962)]; A.A. Kolomenskii, A.N. Lebedev, Sov. Phys. JETP 17179 (1963) [Zh. Eksp. Teor. Fiz. 44261 (1963)]. 
[24] V. Ya. Davydovskii, Sov. Phys. JETP 16629 (1963) [Zh. Eksp. Teor. Fiz. 43886 (1962)].

[25] J. H. Eberly, A. Sleeper, Phys. Rev. 176 (1968), 1570.

[26] J. H. Eberly, Progress in Optics VII. (Ed. E. Wolf) North-Holland, Amsterdam, 1969, pp 359-415; and references therein.

[27] G. Fiore, Acta Appl. Math. 132 (2014), 261.

[28] A. L. Troha et al., Phys. Rev. E 60 (1999), 926.

[29] J. D. Lawson, Eur. J. Phys. 5 (1984), 104; and references therein.

[30] R. B. Palmer, in Frontiers of Particle Beams, Lecture Notes in Physics 296, pp. 607-635, Springer-Verlag, 1988.

[31] E. J. Bochove, G. T. Moore, M. O. Scully, Phys. Rev. A 46, 6640 (1992), appendix A.

[32] R. B. Palmer, AIP Conf. Proc. No. 335 (1995), pp. 90-100; and references therein.

[33] E. Esarey, P. Sprangle, J. Krall Phys. Rev. E 52 (1995), 5443.

[34] G. Fiore, R. Fedele, U. de Angelis, Phys. Plasmas 21 (2014), 113105.

[35] F. X. Kaertner (ed.), Few-Cycle Laser Pulse Generation and Its Applications, Springer, 448 p., 2004; and references therein.

[36] K. Akimoto, J. Phys. Soc. Jpn. 65 (1996), 2020.

[37] A. Couairon, J. Biegertb, C. P. Haurib, W. Kornelisb, F. W. Helbingb, U. Kellerb, A. Mysyrowiczc, J. Mod. Optics 53 (2006), 75-85.

[38] U. Morgner, Nature Photonics 4 (2010), 14-15.

[39] W. Yu et al., Phys. Rev. E 61 (2000), R2220.

[40] A. L. Troha, F. V. Hartemann, Phys. Rev. E 65 (2002), 028502.

[41] P. M. Woodward, J. Inst. Electr. Eng., 93 (1947), 1554.

[42] P. M. Woodward, J. D. Lawson, J. I.E.E. 95, Part III (1948), 363; and references therein.

[43] G. Fiore, On the impact of short laser pulses on cold diluted plasmas, in preparation.

[44] P. A. M. Dirac, Proc. R. Soc. London, Ser. A 167 (1938), 148-169.

[45] V. P. Milant'ev, Physics - Uspekhi 56 (2013), 823-832; and references therein.

[46] L.D. Landau, E.M. Lifshitz, Mechanics, $3^{\text {rd }}$ ed. (translated from the Russian), Pergamon Press, 1976.

[47] G. Fiore, EPJ Web of Conferences Volume 16704004 (2018). 